\title{
Gradient estimates in parabolic problems with unbounded coefficients
}

by

\author{
M. BerToldi (Trento) and S. Fornaro (Lecce)
}

\begin{abstract}
We study, with purely analytic tools, existence, uniqueness and gradient estimates of the solutions to the Neumann problems associated with a second order elliptic operator with unbounded coefficients in spaces of continuous functions in an unbounded open set $\Omega$ in $\mathbb{R}^{N}$.
\end{abstract}

1. Introduction. In this paper we consider a linear second order elliptic operator,

$$
\mathcal{A}=\sum_{i, j=1}^{N} q_{i j} D_{i j}+\sum_{i=1}^{N} F_{i} D_{i}-V,
$$

with regular possibly unbounded coefficients in a regular convex open set $\Omega \subset \mathbb{R}^{N}$. The set $\Omega$ may be unbounded. We study existence, uniqueness and gradient estimates of the solutions to the Neumann problems

$$
\begin{aligned}
& \begin{cases}u_{t}(t, x)-\mathcal{A} u(t, x)=0, & t>0, x \in \Omega, \\
\frac{\partial u}{\partial \nu}(t, x)=0, & t>0, x \in \partial \Omega, \\
u(0, x)=f(x), & x \in \Omega,\end{cases} \\
& \begin{cases}\lambda u(x)-\mathcal{A} u(x)=f(x), & x \in \Omega, \\
\frac{\partial u}{\partial \nu}(x)=0, & x \in \partial \Omega,\end{cases}
\end{aligned}
$$

where $f$ is continuous and bounded and $\nu$ is the outward unit normal vector to $\partial \Omega$. In the case $\Omega=\mathbb{R}^{N}$ problems (1.1) and (1.2) are replaced by similar ones without any boundary condition.

These are classical problems in analysis which are well understood if the coefficients of $\mathcal{A}$ are bounded. On the other hand, in the recent literature the interest towards elliptic operators with unbounded coefficients is growing up. Motivations come from stochastic analysis and change of coordinates that

2000 Mathematics Subject Classification: 35K20, 47D07, 60J35. 
transform elliptic operators with bounded coefficients to elliptic operators with unbounded coefficients. In the case $\Omega=\mathbb{R}^{N}$ several results of existence, uniqueness and regularity are known (see [5], [10], [11], [15], [21] and the overview [17]). Stochastic calculus is a useful tool ([5], [21], [23]); in particular the recent book [5] of Sandra Cerrai contains a deep and exhaustive analysis of what can be proved by stochastic methods.

Our main assumptions are a dissipativity condition on the $\operatorname{drift} F=$ $\left(F_{1}, \ldots, F_{N}\right)$, a Lyapunov type condition ensuring that a maximum principle holds, and that $V$ is bounded from below. Without loss of generality, we may assume that $V(x) \geq 0$ for all $x \in \Omega$. We need other technical assumptions (see Section 2), which are automatically satisfied in the (still important) case $\mathcal{A}=\Delta+\sum F_{i} D_{i}$.

We consider problem (1.1) and we prove that there exists a unique bounded classical solution $u(t, x)$. To do this, we consider the solutions $u_{n}$ of Neumann problems in a nested sequence $\Omega_{n}$ of bounded domains whose union is $\Omega$, and we prove that $u_{n}$ converges to a solution of (1.1). We remark that one could approximate the solution with solutions of suitable mixed boundary value problems in $\Omega_{n}$ in such a way that for nonnegative initial data the approximating sequence is increasing. This was done by Seizo Itô in his pioneering paper [10]. Although this further property could be of much help in some steps, our techniques to get the gradient bounds do not work with such boundary conditions. Therefore we consider the Neumann boundary condition in each $\Omega_{n}$.

If we set $\left(P_{t} f\right)(x)=u(t, x)$, then $P_{t}$ turns out to be a semigroup of linear operators in the space $C_{\mathrm{b}}(\bar{\Omega})$ of continuous and bounded functions in $\bar{\Omega}$. We remark that in general $P_{t}$ is not strongly continuous in $C_{\mathrm{b}}(\bar{\Omega})$ and in its subspace $\operatorname{BUC}(\bar{\Omega})$ of uniformly continuous and bounded functions. This is a typical fact for semigroups associated with elliptic operators with unbounded coefficients. Therefore the generator cannot be defined in the classical way. In the literature there are several alternative definitions of generator; here we consider the weak generator introduced by Enrico Priola in [19].

Our aim is to prove gradient estimates for $P_{t}$. We start by showing that

$$
\begin{array}{ll}
\left|\nabla P_{t} f(x)\right| \leq \frac{C_{T}}{\sqrt{t}}\|f\|_{\infty}, & 0<t<T, x \in \bar{\Omega}, f \in C_{\mathrm{b}}(\bar{\Omega}), \\
\left|\nabla P_{t} f(x)\right| \leq C_{T}\left(\|f\|_{\infty}+\|\nabla f\|_{\infty}\right), & 0<t \leq T, x \in \bar{\Omega}, f \in C_{\nu}^{1}(\bar{\Omega}),
\end{array}
$$
where

$$
C_{\nu}^{1}(\bar{\Omega})=\left\{u \in C_{\mathrm{b}}^{1}(\bar{\Omega}): \frac{\partial u}{\partial \nu}(x)=0, x \in \partial \Omega\right\} .
$$

We prove (1.3) and (1.4) using the Bernstein method, i.e. we apply the maximum principle to the equation satisfied by $z_{n}=u_{n}^{2}+t\left|\nabla u_{n}\right|^{2}$ (respec- 
tively $z_{n}=u_{n}^{2}+\left|\nabla u_{n}\right|^{2}$ ), which gives a bound for $z_{n}$ independent of $n$, and then we obtain (1.3) (respectively (1.4)) letting $n \rightarrow \infty$. We observe that the convexity assumption on $\Omega$ is crucial at this point, since it leads to the condition $\partial z_{n} / \partial \nu \leq 0$ at the boundary (see Lemma 2.4). However, the case of a nonconvex domain will be object of a future investigation by the authors. In the case $\Omega=\mathbb{R}^{N}$ the previous estimates were proved in [15] with the same method and in [5] with probabilistic methods. As a consequence of (1.3), the domain of the weak generator of $P_{t}$ is contained in $C_{\mathrm{b}}^{1}(\bar{\Omega})$.

In Section 4 we assume that $V \equiv 0$ and in the case $q_{i j} \equiv \delta_{i j}$ we prove the estimates

$$
\left|\nabla P_{t} f(x)\right|^{p} \leq e^{k_{0} p t} P_{t}\left(|\nabla f|^{p}\right)(x), \quad t \geq 0, x \in \bar{\Omega}, f \in C_{\nu}^{1}(\bar{\Omega}),
$$

for all $p \geq 1$, where $k_{0} \in \mathbb{R}$ is such that

$$
\sum_{i, j=1}^{N} D_{i} F_{j}(x) \xi_{i} \xi_{j} \leq k_{0}|\xi|^{2}, \quad x \in \Omega, \xi \in \mathbb{R}^{N} .
$$

If the coefficients $q_{i j}$ are not constant we prove the similar estimate

$$
\left|\nabla P_{t} f(x)\right|^{p} \leq e^{\sigma_{p} t} P_{t}\left(|\nabla f|^{p}\right)(x), \quad t \geq 0, x \in \bar{\Omega}, f \in C_{\nu}^{1}(\bar{\Omega}),
$$

for all $p>1$, where $\sigma_{p} \in \mathbb{R}$ is a suitable constant. These estimates have interesting consequences. First, if there exists an invariant measure for $P_{t}$, that is, a probability measure $\mu$ such that

$$
\int_{\Omega} P_{t} f d \mu=\int_{\Omega} f d \mu, \quad t \geq 0, f \in C_{\mathrm{b}}(\bar{\Omega}),
$$

estimates (1.6) and (1.8) are of much help in the study of the realization of $P_{t}$ in the spaces $L^{p}(\Omega, \mu), 1 \leq p<\infty$. (1.6) with $p=1$ and $k_{0}<0$ yields the hypercontractivity of $P_{t}$ in the space $L^{2}(\Omega, \mu)$ and the logarithmic Sobolev inequality. This is the well known Bakry-Émery criterion. (1.8) with $p=2$ and $\sigma_{2}<0$ yields the Poincaré inequality in $L^{2}(\Omega, \mu)$ and the spectral gap for the generator of $P_{t}$ in $L^{2}(\Omega, \mu)$ (see for example [6, Section 10.5]).

Secondly, we deduce the pointwise estimates

$$
\begin{aligned}
& \left|\nabla P_{t} f(x)\right|^{p} \leq\left(\frac{\sigma_{2} \mu_{0}^{-1}}{2\left(1-e^{-\sigma_{2} t}\right)}\right)^{p / 2} P_{t}\left(|f|^{p}\right)(x), \quad t>0, p \geq 2, \\
& \left|\nabla P_{t} f(x)\right|^{p} \leq \frac{c_{p} \sigma_{p} \mu_{0}^{-1}}{t^{p / 2-1}\left(1-e^{-\sigma_{p} t}\right)} P_{t}\left(|f|^{p}\right)(x), \quad t>0,1<p<2,
\end{aligned}
$$

for $f \in C_{\mathrm{b}}(\bar{\Omega})$, where $c_{p}>0$ is a suitable constant. Estimates (1.9) give the optimal constant in (1.3); moreover integrating over $\Omega$ with respect to the invariant measure $\mu$ we get the corresponding estimates for $\nabla P_{t} f$ in $L^{p}(\Omega, \mu)$, when $f \in L^{p}(\Omega, \mu)$. 
In the case $\Omega=\mathbb{R}^{N}$, estimate (1.6) and estimate (1.9) with $p=2$ were proved respectively in [2] and [3] in the setting of abstract Markov generators, for functions belonging to a suitable algebra of smooth functions which is required to be invariant under the generator. Estimate (1.6) was also proved in [23] by probabilistic methods. A probabilistic approach is used in [20] as well for establishing estimate (1.6) in the case of a compact Riemannian manifold with convex boundary or of a complete manifold without boundary.

Dissipativity conditions of the type (1.7) are of crucial importance to get gradient estimates. Indeed, in Section 5 we give a counterexample to estimate (1.3) for an operator $\mathcal{A}=\Delta+\sum F_{i} D_{i}$ where $F$ does not satisfy (1.7). Concerning estimate (1.6), in the case of variable coefficients $q_{i j}$ the constant $\sigma_{p}$ blows up as $p \rightarrow 1$, and we do not expect that (1.6) also holds for $p=1$. Estimate (1.9) too fails in general for $p=1$, as we show in the case of the heat semigroup. Finally we show an example related with the Ornstein-Uhlenbeck operator.

Acknowledgements. The authors would like to thank Alessandra Lunardi and Giorgio Metafune for many useful discussions and comments.

Notations. For $x \in \mathbb{R}^{N},|x|$ denotes the euclidean norm, and $B_{R}=$ $\left\{x \in \mathbb{R}^{N}:|x|<R\right\}$ denotes the open ball with radius $R>0$. We denote by $C_{\mathrm{b}}(\bar{\Omega})$ the space of bounded continuous functions in $\bar{\Omega}$ and by $C_{\mathrm{b}}^{k}(\bar{\Omega})$ the space of functions with derivatives up to order $k$ in $C_{\mathrm{b}}(\bar{\Omega}) . C_{0}(\Omega)$ is the space of functions in $C_{\mathrm{b}}(\bar{\Omega})$ vanishing at $\partial \Omega$ and at infinity. For $0<\alpha \leq 1$ and $a<b, C^{k+\alpha}(\bar{\Omega})$ is the space of functions such that the derivatives of order $k$ are $\alpha$-Hölder continuous in $\bar{\Omega}$, and $C^{1+\alpha / 2,2+\alpha}([a, b] \times \bar{\Omega})$ is the space of functions $u=u(t, x)$ such that $D_{t} u$ and $D_{i j} u$ are $\alpha$-Hölder continuous in $[a, b] \times \bar{\Omega}$ with respect to the parabolic distance $d((t, x),(s, y))=|t-s|^{1 / 2}$ $+|x-y|$. By $C_{\mathrm{loc}}^{1+\alpha / 2,2+\alpha}(] 0, \infty[\times \bar{\Omega})$ we mean the space of functions $u$ such that $u \in C^{1+\alpha / 2,2+\alpha}\left([\varepsilon, T] \times \overline{\Omega^{\prime}}\right)$ for all $0<\varepsilon<T$ and bounded open $\Omega^{\prime} \subseteq \Omega$. Analogously $C_{\text {loc }}^{1+\alpha}(\bar{\Omega})$ denotes the space of functions which belong to $C^{1+\alpha}\left(\overline{\Omega^{\prime}}\right)$ for all bounded open $\Omega^{\prime} \subseteq \Omega$.

We use the notation

for all $\xi \in \mathbb{R}^{N}$ and $x \in \Omega$.

$$
\mathcal{Q}[\xi](x)=\sum_{i, j=1}^{N} q_{i j}(x) \xi_{i} \xi_{j}
$$

2. Preliminary results. First we state our assumptions that will be kept throughout the paper. $\Omega \subset \mathbb{R}^{N}$ is a convex open set with $C^{2+\alpha}$ boundary. The coefficients of the operator $\mathcal{A}$ are real-valued, belong to $C_{\text {loc }}^{1+\alpha}(\bar{\Omega})$ 
and satisfy the following conditions:

$$
\begin{aligned}
& q_{i j}=q_{j i}, \quad \sum_{i, j=1}^{N} q_{i j}(x) \xi_{i} \xi_{j} \geq \mu(x)|\xi|^{2}, \quad x \in \Omega, \quad \xi \in \mathbb{R}^{N}, \\
& \inf _{x \in \Omega} \mu(x)=\mu_{0}>0, \\
& \left|\nabla q_{i j}(x)\right| \leq M \mu(x), \quad x \in \Omega, i, j=1, \ldots, N, \\
& \sum_{i, j=1}^{N} D_{i} F_{j}(x) \xi_{i} \xi_{j} \leq\left(\beta V(x)+k_{0}\right)|\xi|^{2}, \quad x \in \Omega, \quad \xi \in \mathbb{R}^{N}, \\
& V(x) \geq 0, \quad|\nabla V(x)| \leq \gamma(1+V(x)), \quad x \in \Omega,
\end{aligned}
$$

for some constants $M, \gamma \geq 0, k_{0}, \beta \in \mathbb{R}, \beta<1 / 2$. Moreover, we suppose that there exist a positive function $\varphi \in C^{2}(\bar{\Omega})$ and $\lambda_{0}>0$ such that

$$
\lim _{|x| \rightarrow \infty} \varphi(x)=\infty, \quad \sup _{\Omega}\left(\mathcal{A} \varphi-\lambda_{0} \varphi\right)<\infty, \quad \frac{\partial \varphi}{\partial \nu}(x) \geq 0, \quad x \in \partial \Omega .
$$

We introduce the following realization of the operator $\mathcal{A}$ with homogeneous Neumann boundary condition:

$$
\begin{aligned}
& D(\mathcal{A})=\left\{u \in C_{\mathrm{b}}(\bar{\Omega}) \cap W^{2, p}\left(\Omega \cap B_{R}\right) \text { for all } R>0, p<\infty:\right. \\
& \left.\qquad \mathcal{A} u \in C_{\mathrm{b}}(\bar{\Omega}), \frac{\partial u}{\partial \nu}(x)=0, x \in \partial \Omega\right\} .
\end{aligned}
$$

We remark that if $\Omega=\mathbb{R}^{N}$ our results can be generalized to operators with locally Hölder continuous coefficients satisfying suitable assumptions by a standard convolution approximation (see Remark 4.4).

In this section we collect some preliminary results which are the main tools for the study of problems (1.1) and (1.2). We start by proving maximum principles for such problems, and consequent uniqueness results.

Proposition 2.1. Let $\left.\left.\left.z \in C([0, T] \times \bar{\Omega}) \cap C^{0,1}(] 0, T\right] \times \bar{\Omega}\right) \cap C^{1,2}(] 0, T\right] \times$ $\Omega)$ be a bounded function satisfying

$$
\begin{cases}z_{t}(t, x)-\mathcal{A} z(t, x) \leq 0, & 0<t \leq T, x \in \Omega, \\ \frac{\partial z}{\partial \nu}(t, x) \leq 0, & 0<t \leq T, x \in \partial \Omega \\ z(0, x) \leq 0, & x \in \Omega .\end{cases}
$$

Then $z \leq 0$. In particular there exists at most one bounded classical solution of problem (1.1).

Proof. We may suppose that $\mathcal{A} \varphi-\lambda_{0} \varphi \leq 0$; otherwise we replace $\varphi$ by $\varphi+C$ for a suitable constant $C>0$. Set $v(t, \bar{x})=e^{-\lambda_{0} t} z(t, x)$; we prove that 
$v \leq 0$, then the statement follows. We consider the sequence

$$
v_{n}(t, x)=v(t, x)-\frac{1}{n} \varphi(x), \quad 0 \leq t \leq T, x \in \Omega,
$$

and we observe that

$$
\begin{cases}D_{t} v_{n}(t, x)-\left(\mathcal{A}-\lambda_{0}\right) v_{n}(t, x) \leq 0, & 0<t \leq T, x \in \Omega, \\ \frac{\partial v_{n}}{\partial \nu}(t, x) \leq 0, & 0<t \leq T, x \in \partial \Omega, \\ v_{n}(0, x) \leq 0, & x \in \bar{\Omega} .\end{cases}
$$

For every $n \in \mathbb{N}$ the function $v_{n}$ attains its maximum in $[0, T] \times \bar{\Omega}$ at some point $\left(t_{n}, x_{n}\right)$. If $t_{n}>0$ and $x_{n} \in \Omega$ then

$$
D_{t} v_{n}\left(t_{n}, v_{n}\right) \geq 0, \quad \mathcal{A} v_{n}\left(t_{n}, x_{n}\right)+V\left(x_{n}\right) v_{n}\left(t_{n}, x_{n}\right) \leq 0,
$$

and consequently, using the equation

$$
\left(\lambda_{0}+V\left(x_{n}\right)\right) v_{n}\left(t_{n}, x_{n}\right) \leq\left(\lambda_{0}+D_{t}-\mathcal{A}\right) v_{n}\left(t_{n}, x_{n}\right) \leq 0 .
$$

Since $\lambda_{0}>0$ this implies that $v_{n}\left(t_{n}, x_{n}\right) \leq 0$.

If $t_{n}=0$ we immediately have $v_{n}\left(t_{n}, x_{n}\right) \leq 0$. Finally, it is not possible that $t_{n}>0$ and $x_{n} \in \partial \Omega$ without any interior maximum point because of the strong maximum principle ([7, Theorem 2.14]).

Therefore we have proved that $v(t, x) \leq n^{-1} \varphi(x)$ for all $0 \leq t \leq T$ and $x \in \bar{\Omega}$. Thus letting $n \rightarrow \infty$ we conclude that $v \leq 0$, as claimed.

The following strong maximum principle is well known for $C^{2}$ solutions.

Proposition 2.2. Let $u \in C_{\mathrm{b}}(\bar{\Omega}) \cap W^{2, p}\left(\Omega \cap B_{R}\right)$ for all $R>0$ and $p<\infty$, and suppose that $\mathcal{A} u \in C_{\mathrm{b}}(\bar{\Omega})$ and

$$
\lambda u(x)-\mathcal{A} u(x) \leq 0, \quad x \in \Omega,
$$

for some $\lambda>0$. Let $x_{0} \in \partial \Omega$ be such that $u\left(x_{0}\right)>0$ and $u(x)<u\left(x_{0}\right)$ for all $x \in \Omega$. Then

$$
\frac{\partial u}{\partial \nu}\left(x_{0}\right)>0
$$

Proof. We follow the proof of the classical Hopf maximum principle (see e.g. [9, Lemma 3.4]). Consider a ball $B(y, r) \subset \Omega$ such that $\bar{B}(y, r) \cap \partial \Omega$ $=\left\{x_{0}\right\}$ and assume that $u>0$ in $B(y, r)$. It is readily seen that there exists $\alpha>0$ such that the function $z(x)=e^{-\alpha|x-y|^{2}}-e^{-\alpha r^{2}}$ satisfies $\mathcal{A} z>0$ in $D=B(y, r) \backslash \bar{B}(y, r / 2)$. Set $w=u+\varepsilon z$, where $\varepsilon>0$ is chosen in such a way that $w(x)<u\left(x_{0}\right)$ for all $x \in \partial B(y, r / 2)$. Then $w(x) \leq u\left(x_{0}\right)$ in $\partial D$ and

$$
\mathcal{A} w(x)=\mathcal{A} u(x)+\varepsilon \mathcal{A} z(x)>\lambda u(x)>0, \quad x \in D .
$$

Let $\bar{x} \in \bar{D}$ be the maximum point of $w$ in $\bar{D}$. It is not possible that $\bar{x} \in D$, otherwise from [17, Lemma 3.2] we should have $\mathcal{A} w(\bar{x}) \leq 0$, which is in 
contradiction with (2.7). Then $\bar{x} \in \partial D$ and necessarily $\bar{x}=x_{0}$. It follows that

$$
\frac{\partial w}{\partial \nu}\left(x_{0}\right)=\frac{\partial u}{\partial \nu}\left(x_{0}\right)+\varepsilon \frac{\partial z}{\partial \nu}\left(x_{0}\right) \geq 0 .
$$

Since $\frac{\partial z}{\partial \nu}\left(x_{0}\right)<0$, this implies $(2.6)$.

Proposition 2.3. Let $u \in C_{\mathrm{b}}(\bar{\Omega}) \cap W^{2, p}\left(\Omega \cap B_{R}\right)$ for all $R>0$ and $p<\infty$, and suppose that $\mathcal{A} u \in C_{\mathrm{b}}(\bar{\Omega})$ and

$$
\begin{cases}\lambda u(x)-\mathcal{A} u(x) \leq 0, & x \in \Omega, \\ \frac{\partial u}{\partial \nu}(x) \leq 0, & x \in \partial \Omega,\end{cases}
$$

for some $\lambda \geq \lambda_{0}$. Then $u \leq 0$.

Proof. As in Proposition 2.1, we may assume that $\mathcal{A} \varphi-\lambda_{0} \varphi \leq 0$. We introduce the sequence

$$
u_{n}(x)=u(x)-\frac{1}{n} \varphi(x), \quad x \in \Omega,
$$

and we note that

$$
\begin{cases}\lambda u_{n}(x)-\mathcal{A} u_{n}(x) \leq 0, & x \in \Omega, \\ \frac{\partial u_{n}}{\partial \nu}(x) \leq 0, & x \in \partial \Omega .\end{cases}
$$

We prove that $u_{n} \leq 0$ for all $n \in \mathbb{N}$; then the conclusion follows by letting $n \rightarrow \infty$. Each $u_{n}$ has a maximum point $x_{n} \in \bar{\Omega}$. If $x_{n} \in \Omega$, then from [17, Lemma 3.2] it follows that $\mathcal{A} u_{n}\left(x_{n}\right) \leq 0$ and, by $(2.9), u_{n}\left(x_{n}\right) \leq 0$. Now assume that $x_{n} \in \partial \Omega$ and $u_{n}(x)<u_{n}\left(x_{n}\right)$ for all $x \in \Omega$ (otherwise there would exist an interior maximum point and we could apply the previous step). Then from Proposition 2.2 and (2.9) it follows that $u_{n}\left(x_{n}\right) \leq 0$, and this completes the proof.

If $\Omega$ is bounded, the above maximum principle for $W^{2, p}$ solutions is well known (see for example [1], [4]).

The following lemma is of crucial importance for our estimates; it holds for convex domains and this is the reason why we have assumed that $\Omega$ is convex.

LEMMA 2.4. Let $\Lambda$ be a convex open set with $C^{1}$ boundary, not necessarily bounded. Let $u \in C^{2}(\bar{\Lambda})$ be such that $\partial u / \partial \nu(x)=0$ for all $x \in \partial \Lambda$. Then the function $v:=|\nabla u|^{2}$ satisfies

$$
\frac{\partial v}{\partial \nu}(x) \leq 0, \quad x \in \partial \Lambda
$$

Proof. Since $\Omega$ is convex, we have $\tau \cdot \frac{\partial \nu}{\partial \tau}(x) \geq 0$ for all $x \in \partial \Omega$ and all vectors $\tau$ tangent to $\partial \Omega$ at $x$ (see [8, Section V.B]). By assumption, 
$\nabla u(x) \cdot \nu(x)=0$ for all $x \in \partial \Omega$ and then differentiating we get

$$
\frac{\partial}{\partial \tau}(\nabla u(x) \cdot \nu(x))=D^{2} u(x) \tau \cdot \nu(x)+\nabla u(x) \cdot \frac{\partial \nu}{\partial \tau}(x)=0, \quad x \in \partial \Lambda,
$$

for every vector $\tau$ tangent to $\partial \Omega$. For $\tau=\nabla u(x)$ we have

$$
\frac{\partial v}{\partial \nu}(x)=2 D^{2} u(x) \tau \cdot \nu(x)=-2 \tau \cdot \frac{\partial \nu}{\partial \tau}(x) \leq 0, \quad x \in \partial \Omega
$$

Now we recall some known results about Neumann problems in bounded domains. Let $\Lambda$ be a bounded open set in $\mathbb{R}^{N}$ with $C^{2+\alpha}$ boundary. Consider the realization of the operator $\mathcal{A}$ in $C(\bar{\Lambda})$ with homogeneous Neumann boundary condition

$$
\begin{aligned}
& D_{\nu}(A)=\left\{u \in W^{2, p}(\Lambda) \text { for all } p<\infty:\right. \\
& \left.\qquad \mathcal{A} u \in C(\bar{\Lambda}), \frac{\partial u}{\partial \nu}(x)=0, x \in \partial \Lambda\right\},
\end{aligned}
$$

and $A u=\mathcal{A} u$ for all $u \in D_{\nu}(A)$. The operator $\left(A, D_{\nu}(A)\right)$ is the generator of a strongly continuous analytic positive contraction semigroup $(S(t))_{t \geq 0}$ in $C(\bar{\Lambda})$ (see e.g. [14, Section 3.1.5]). This means that for all $f \in C(\bar{\Lambda})$ the function $u(t, x)=(S(t) f)(x)$ has the following properties:

(i) $u \in C\left(\left[0, \infty[; C(\bar{\Lambda})) \cap C^{1+\alpha / 2,2+\alpha}([\varepsilon, T] \times \bar{\Lambda})\right.\right.$ for all $0<\varepsilon<T<\infty$;

(ii) $D_{i} u \in C^{1+\alpha / 2,2+\alpha}\left([\varepsilon, T] \times \bar{\Lambda}_{1}\right)$ for all $i=1, \ldots, N, 0<\varepsilon<T<\infty$ and open sets $\Lambda_{1}$ with $\bar{\Lambda}_{1} \subset \Lambda$, in particular $u \in C^{1,3}(] 0, \infty[\times \Lambda)$;

(iii) $u$ is the unique classical bounded solution of the Neumann problem

$$
\begin{cases}D_{t} u(t, x)-A u(t, x)=0, & t>0, x \in \Lambda, \\ \frac{\partial u}{\partial \nu}(t, x)=0, & t>0, x \in \partial \Lambda, \\ u(0, x)=f(x), & x \in \Lambda .\end{cases}
$$

Next we prove a gradient estimate for $S(t) f$, using Bernstein's method (see [15, Theorem 2.4]). It is worth observing that since $\Lambda$ is bounded, this result is well known. Actually, our interest is not in the estimate itself but rather in the fact that the constant $C_{T}$ in (2.12) below does not depend on the domain $\Lambda$ when it is convex. This will be an important step in the study of problem (1.1).

Proposition 2.5. Let $\Lambda$ be a bounded convex open set with $C^{2+\alpha}$ boundary. For all fixed $T>0$ there exists a constant $C_{T}>0$ independent of $\Lambda$ such that

$$
|\nabla S(t) f(x)| \leq \frac{C_{T}}{\sqrt{t}}\|f\|_{\infty}, \quad 0<t \leq T, x \in \bar{\Lambda},
$$

for every $f \in C(\bar{\Lambda})$. 
Proof. We may suppose that $V \geq 1$; the general case follows by considering the operator $A^{\prime}=A-I$. Assume first that $f \in D_{\nu}(A)$; set $u(t, x)=$ $(S(t) f)(x)$ and define the function

$$
v(t, x)=u^{2}(t, x)+a t|\nabla u(t, x)|^{2}, \quad t \geq 0, x \in \Lambda,
$$

where $a>0$ is a parameter that will be chosen later. Then $\left.\left.v \in C^{1,2}(] 0, T\right] \times \Lambda\right)$ $\left.\left.\cap C^{0,1}(] 0, T\right] \times \bar{\Lambda}\right)$; moreover, since $f \in D_{\nu}(A)$, we have $u \in C\left([0, T] ; D_{\nu}(A)\right)$; in particular $\nabla u \in C([0, T] \times \bar{\Lambda})$ and then $v \in C([0, T] \times \bar{\Lambda})$.

We claim that for a suitable value of $a>0$ independent of $\Lambda$, we have

$$
\begin{aligned}
v_{t}(t, x)-\mathcal{A} v(t, x) \leq 0, & 0<t<T, x \in \Lambda, \\
\frac{\partial v}{\partial \nu}(t, x) \leq 0, & 0<t<T, x \in \partial \Lambda ;
\end{aligned}
$$

then the maximum principle implies

$$
v(t, x) \leq \sup _{x \in \bar{\Lambda}} v(0, x)=\|f\|_{\infty}^{2}, \quad 0 \leq t \leq T, x \in \bar{\Lambda},
$$

which yields (2.12) with $C_{T}=a^{-1 / 2}$.

The boundary condition (2.14) follows from Lemma 2.4. For (2.13), a straightforward computation shows that $v$ satisfies the equation

where

$$
\begin{aligned}
v_{t}(t, x)-\mathcal{A} v(t, x)= & a|\nabla u(t, x)|^{2}-2 \sum_{i, j=1}^{N} q_{i j}(x) D_{i} u(t, x) D_{j} u(t, x) \\
& +g_{1}(t, x)+g_{2}(t, x),
\end{aligned}
$$

$$
\begin{aligned}
g_{1}(t, x)= & 2 a t \sum_{i, j=1}^{N} D_{i} F_{j}(x) D_{i} u(t, x) D_{j} u(t, x)-a t V(x)|\nabla u(t, x)|^{2} \\
& -2 a t u(t, x) \nabla u(t, x) \cdot \nabla V(x)-V(x) u^{2}(t, x), \\
g_{2}(t, x)= & 2 a t\left(\sum_{i, j, k=1}^{N} D_{k} q_{i j}(x) D_{k} u(t, x) D_{i j} u(t, x)\right. \\
& \left.-\sum_{i, j, k=1}^{N} q_{i j}(x) D_{i k} u(t, x) D_{j k} u(t, x)\right) .
\end{aligned}
$$

Let us estimate the function $g_{1}$. Using (2.3), (2.4) and recalling that $V \geq 1$ we get, for all $\varepsilon>0$,

$$
\begin{aligned}
g_{1}(t, x) \leq & 2 a t\left(\beta V+k_{0}\right)|\nabla u|^{2}-a t V|\nabla u|^{2} \\
& +2 a \gamma C_{\varepsilon} t(1+V)|u|^{2}+2 a \gamma \varepsilon t(1+V)|\nabla u|^{2}-V u^{2} \\
\leq & a t(2 \beta-1+2 \gamma \varepsilon) V|\nabla u|^{2}+\left(4 a \gamma C_{\varepsilon} t-1\right) V u^{2}+2 a t\left(k_{0}+\gamma \varepsilon\right)|\nabla u|^{2},
\end{aligned}
$$

where $C_{\varepsilon}>0$ is a constant. Since $\beta<1 / 2$ we can choose $\varepsilon=\varepsilon(\beta, \gamma)$ such 
that $2 \beta-1+2 \gamma \varepsilon<0$ and we get

$$
g_{1}(t, x) \leq\left(4 a \gamma C_{\varepsilon} t-1\right) V u^{2}+2 a t\left(k_{0}+\gamma \varepsilon\right)|\nabla u|^{2} .
$$

Concerning $g_{2}$, from (2.2) we have

$$
\begin{aligned}
\sum_{i, j, k=1}^{N} D_{k} q_{i j} D_{k} u D_{i j} u & \leq M \mu(x) \sum_{k=1}^{N}\left|D_{k} u\right| \sum_{i, j=1}^{N}\left|D_{i j} u\right| \\
& \leq M N^{3 / 2} \mu(x)|\nabla u|\left(\sum_{i, j=1}^{N}\left(D_{i j} u\right)^{2}\right)^{1 / 2} \\
& \leq \mu(x) \sum_{i, j=1}^{N}\left(D_{i j} u\right)^{2}+\frac{1}{4} M^{2} N^{3} \mu(x)|\nabla u|^{2}
\end{aligned}
$$

and therefore

$$
\begin{aligned}
g_{2}(t, x) \leq & 2 a t\left(\mu(x) \sum_{i, j=1}^{N}\left(D_{i j} u\right)^{2}\right. \\
& \left.+\frac{1}{4} M^{2} N^{3} \mu(x)|\nabla u|^{2}-\mu(x) \sum_{i, j=1}^{N}\left(D_{i j} u\right)^{2}\right) \\
= & \frac{1}{2} a t M^{2} N^{3} \mu(x)|\nabla u|^{2} .
\end{aligned}
$$

Estimates (2.15) and (2.16) imply that

$$
\begin{aligned}
v_{t}(t, x)- & \mathcal{A} v(t, x) \\
\leq & \left\{a+2 a t\left(k_{0}+\gamma \varepsilon\right)+\left(\frac{1}{2} a t M^{2} N^{3}-2\right) \mu(x)\right\}|\nabla u(t, x)|^{2} \\
& +\left(4 a \gamma C_{\varepsilon} t-1\right) V(x) u^{2}(t, x) \\
\leq & \left\{a+2 a T\left(k_{0}^{+}+\gamma \varepsilon\right)+\left(\frac{1}{2} a T M^{2} N^{3}-2\right) \mu(x)\right\}|\nabla u(t, x)|^{2} \\
& +\left(4 a \gamma C_{\varepsilon} T-1\right) V(x) u^{2}(t, x)
\end{aligned}
$$

for all $t \in] 0, T]$ and $x \in \Lambda$. It is clear now that there exists a sufficiently small value $a>0$ which depends on $\mu_{0}, M, k_{0}, \beta, \gamma, N, T$ but not on $\Lambda$ such that (2.13) holds.

If $f \in C(\bar{\Lambda})$ the statement follows easily from the semigroup law, since $S(t)$ is analytic:

$$
\begin{aligned}
|\nabla S(t) f(x)| & =|\nabla S(t / 2) S(t / 2) f(x)| \\
& \leq \frac{\sqrt{2} C_{T}}{\sqrt{t}}\|S(t / 2) f\|_{\infty} \leq \frac{\sqrt{2} C_{T}}{\sqrt{t}}\|f\|_{\infty} .
\end{aligned}
$$


3. Construction of the associated semigroup. In this section we prove that there exist bounded solutions to problems (1.1) and (1.2), we show that there exists a semigroup $\left(P_{t}\right)_{t \geq 0}$ in $C_{\mathrm{b}}(\bar{\Omega})$ which yields the solution of (1.1), and we study the main properties of $P_{t}$.

We consider a nested sequence $\left\{\Omega_{n}\right\}_{n \in \mathbb{N}}$ of convex bounded open sets with $C^{2+\alpha}$ boundary such that

$$
\bigcup_{n \in \mathbb{N}} \Omega_{n}=\Omega, \quad \partial \Omega \subset \bigcup_{n \in \mathbb{N}} \partial \Omega_{n} .
$$

We define the domain of the realization of $\mathcal{A}$ in $\Omega_{n}$ by

$$
\begin{aligned}
& D_{n}(\mathcal{A})=\left\{u \in W^{2, p}\left(\Omega_{n}\right) \text { for all } p<\infty:\right. \\
& \left.\qquad \mathcal{A} u \in C\left(\bar{\Omega}_{n}\right), \frac{\partial u}{\partial \nu}(x)=0, x \in \partial \Omega_{n}\right\},
\end{aligned}
$$

and we denote the associated semigroup by $\left(T_{n}(t)\right)_{t \geq 0}$. Here is the existence theorem for problem (1.1).

THEOREM 3.1. For every $f \in C_{\mathrm{b}}(\bar{\Omega})$ there exists a unique bounded solution $u(t, x)$ of problem $(1.1)$ belonging to $C\left(\left[0, \infty[\times \bar{\Omega}) \cap C_{\mathrm{loc}}^{1+\alpha / 2,2+\alpha}(] 0, \infty[\right.\right.$ $\times \bar{\Omega})$. Moreover

$$
u(t, x)=\lim _{n \rightarrow \infty}\left(T_{n}(t)\right) f(x), \quad t \geq 0, x \in \bar{\Omega} .
$$

If we set $P_{t} f=u(t, \cdot)$, then $\left(P_{t}\right)_{t \geq 0}$ is a positive contraction semigroup in $C_{\mathrm{b}}(\bar{\Omega})$. Moreover

$$
\left\|\nabla P_{t} f\right\|_{\infty} \leq \frac{C_{T}}{\sqrt{t}}\|f\|_{\infty}, \quad 0<t \leq T,
$$

where $C_{T}$ is as in (2.12).

Proof. Set $u_{n}(t, x)=\left(T_{n}(t) f\right)(x)$. Let $\Omega^{\prime} \subset \Omega$ be a bounded open set and $0<\varepsilon<T$. From [13, Theorem IV.10.1] it follows that if $\Omega^{\prime \prime} \subset \Omega$ is a bounded open set such that $\Omega^{\prime} \subset \Omega^{\prime \prime}$ and $\operatorname{dist}\left(\Omega^{\prime}, \Omega \backslash \Omega^{\prime \prime}\right)>0$, then there exists a constant $C=C\left(\varepsilon, T, \Omega^{\prime}, \Omega^{\prime \prime}\right)>0$ such that

$$
\left\|u_{n}\right\|_{C^{1+\alpha / 2,2+\alpha}\left([\varepsilon, T] \times \overline{\Omega^{\prime}}\right)} \leq C\left\|u_{n}\right\|_{C\left([0, T] \times \overline{\Omega^{\prime \prime}}\right)} .
$$

Hence

$$
\left\|u_{n}\right\|_{C^{1+\alpha / 2,2+\alpha}\left([\varepsilon, T] \times \overline{\Omega^{\prime}}\right)} \leq C\|f\|_{\infty}
$$

for all $n \in \mathbb{N}$ such that $\Omega^{\prime \prime} \subset \Omega_{n}$, and therefore the sequence $\left(u_{n}\right)_{n \in \mathbb{N}}$ is relatively compact in $C^{1,2}\left([\varepsilon, T] \times \overline{\Omega^{\prime}}\right)$. Considering an increasing sequence of domains $\left[\varepsilon_{n}, T_{n}\right] \times \overline{\Omega_{n}^{\prime}}$ whose union is $] 0, \infty[\times \bar{\Omega}$ and using a diagonal procedure we can conclude that there exists a subsequence $\left(u_{n_{k}}\right)_{k \in \mathbb{N}}$ (possibly depending on $f$ ) such that

$$
\lim _{k \rightarrow \infty} u_{n_{k}}(t, x)=u(t, x), \quad t>0, x \in \bar{\Omega},
$$


where $u \in C_{\text {loc }}^{1+\alpha / 2,2+\alpha}(] 0, \infty[\times \bar{\Omega})$. Moreover $\left(u_{n_{k}}\right)_{k \in \mathbb{N}}$ converges to $u$ in $C^{1,2}\left([\varepsilon, T] \times \overline{\Omega^{\prime}}\right)$ for all $0<\varepsilon<T$ and for all bounded open sets $\Omega^{\prime} \subset \Omega$.

We prove that $u$ is a bounded classical solution of problem (1.1). The function $u$ is a solution of the equation $u_{t}-\mathcal{A} u=0$ in $] 0, \infty[\times \Omega$. This follows by letting $k \rightarrow \infty$ in the equation satisfied by $u_{n_{k}}$. Moreover since

$$
|u(t, x)| \leq\|f\|_{\infty}, \quad t>0, x \in \bar{\Omega},
$$

we see that $u$ is bounded in $] 0, \infty[\times \bar{\Omega}$. The boundary condition

$$
\frac{\partial u}{\partial \nu}(t, x)=0, \quad t>0, x \in \partial \Omega,
$$

follows immediately since $u_{n_{k}}$ converges to $u$ in $C^{1,2}\left([\varepsilon, T] \times \overline{\Omega^{\prime}}\right)$ for all $0<$ $\varepsilon<T$ and $\Omega^{\prime} \subset \Omega$ a bounded open set. Finally we prove that $u$ is continuous at $\left(0, x_{0}\right)$ with value $f\left(x_{0}\right)$ for all $x_{0} \in \bar{\Omega}$. Consider two neighborhoods $U_{1} \subset U_{0}$ of $x_{0}$. Set $\Omega_{0}=U_{0} \cap \Omega$ and $\Omega_{1}=U_{1} \cap \Omega$ and suppose that $\Omega_{0}$ is convex and has $C^{2+\alpha}$ boundary. Let $\theta \in C^{\infty}\left(\bar{\Omega}_{0}\right)$ be such that $\theta=0$ in a neighborhood of $\Omega \cap \partial U_{0}, \theta=1$ in $\bar{\Omega}_{1}$ and $\partial \theta / \partial \nu=0$ in $U_{0} \cap \partial \Omega$. Define

$$
v_{n}(t, x)=\theta(x) u_{n}(t, x), \quad t>0, x \in \Omega_{0} .
$$

Then $v_{n}$ satisfies the boundary condition

$$
\frac{\partial v_{n}}{\partial \nu}(t, x)=\theta(x) \frac{\partial u_{n}}{\partial \nu}(t, x)+u_{n}(t, x) \frac{\partial \theta}{\partial \nu}(x)=0
$$

for all $t>0$ and $x \in \partial \Omega_{0}$ and for all $n$ such that $\Omega_{0} \subset \Omega_{n}$. Moreover $v_{n}$ satisfies the equation

$$
D_{t} v_{n}(t, x)-\mathcal{A} v_{n}(t, x)=\psi_{n}(t, x), \quad t>0, x \in \Omega_{0},
$$

where

$$
\psi_{n}(t, x)=-u_{n}(t, x)(\mathcal{A}+V(x)) \theta(x)-2 \sum_{i, j=1}^{N} q_{i j}(x) D_{i} u_{n}(t, x) D_{j} \theta(x) .
$$

Since $T_{n}(t)$ satisfies the gradient estimate (2.12), it follows that there exists a constant $C>0$ such that

$$
\left\|\psi_{n}(t)\right\|_{\infty} \leq \frac{C}{\sqrt{t}}, \quad 0<t \leq T
$$

for all $n \in \mathbb{N}$. Let $T(t)$ be the strongly continuous analytic semigroup generated by the realization of $\mathcal{A}$ in $C\left(\bar{\Omega}_{0}\right)$ with Neumann boundary conditions. From [14, Proposition 4.1.2] it follows that $v_{n}(t)$ can be written as

$$
v_{n}(t)=T(t)(\theta f)+\int_{0}^{t} T(t-s) \psi_{n}(s) d s .
$$


Since $v_{n}=u_{n}$ in $\bar{\Omega}_{1}$, if $\left.(t, x) \in\right] 0, T\left[\times \bar{\Omega}_{1}\right.$ we have

$$
\left|u_{n_{k}}(t, x)-f\left(x_{0}\right)\right| \leq\left|T(t)(\theta f)(x)-f\left(x_{0}\right)\right|+\int_{0}^{t}\left\|T(t-s) \psi_{n_{k}}(s)\right\|_{\infty} d s .
$$

Using (3.6) and letting $k \rightarrow \infty$ we get

$$
\left|u(t, x)-f\left(x_{0}\right)\right| \leq\left|T(t)(\theta f)(x)-f\left(x_{0}\right)\right|+\int_{0}^{t} \frac{C}{\sqrt{s}} d s,
$$

which shows that $u$ is continuous at $\left(0, x_{0}\right)$. Since $x_{0} \in \bar{\Omega}$ is arbitrary, we conclude that $u$ is continuous in $[0, T] \times \bar{\Omega}$. Thus we have proved that $u$ is a bounded classical solution of problem (1.1).

We claim that the whole sequence $\left(u_{n}\right)_{n \in \mathbb{N}}$ converges to $u$ in $C^{1,2}([\varepsilon, T]$ $\left.\times \overline{\Omega^{\prime}}\right)$ for all $0<\varepsilon<T$ and $\Omega^{\prime} \subset \Omega$ a bounded open set. Indeed, consider any subsequence $\left(u_{n_{k}}\right)_{k \in \mathbb{N}}$ of $\left(u_{n}\right)_{n \in \mathbb{N}}$. The previous argument can be applied to $\left(u_{n_{k}}\right)_{k \in \mathbb{N}}$ and it follows that there is a subsequence $\left(u_{n_{k_{j}}}\right)_{j \in \mathbb{N}}$ and a function $v$ such that $v$ is a classical bounded solution of problem (1.1) and $\left(u_{n_{k_{j}}}\right)_{j \in \mathbb{N}}$ converges to $v$. But from Proposition 2.1 it follows that $u=v$. This shows that the whole sequence converges to $u$.

Writing $\left(P_{t} f\right)(x)=u(t, x)$, we get the positivity of $P_{t}$ directly from the positivity of $T_{n}(t)$. The semigroup law for the linear operators $P_{t}$ follows in a standard way from uniqueness.

Finally, according to Proposition 2.5, for all $T>0$ there exists a constant $C_{T}>0$ such that

$$
\left|\nabla T_{n}(t) f(x)\right| \leq \frac{C_{T}}{\sqrt{t}}\|f\|_{\infty}, \quad 0<t \leq T, x \in \bar{\Omega}_{n},
$$

for all $n \in \mathbb{N}$. Letting $n \rightarrow \infty$ we get (3.3).

The next proposition shows some continuity properties of $P_{t}$ that will be useful subsequently.

Proposition 3.2. If $\left(f_{n}\right)_{n \in \mathbb{N}} \subset C_{\mathrm{b}}(\bar{\Omega})$ is a bounded sequence which converges pointwise in $\Omega$ to a function $f \in C_{\mathrm{b}}(\bar{\Omega})$, then $\left(P_{t} f_{n}\right)(x)$ converges to $\left(P_{t} f\right)(x)$ in $C^{1,2}\left([\varepsilon, T] \times \overline{\Omega^{\prime}}\right)$ for all $0<\varepsilon<T$ and all bounded sets $\Omega^{\prime} \subset \Omega$. If $\left(f_{n}\right)$ converges to $f$ uniformly on compact subsets of $\bar{\Omega}$, then $\left(P_{t} f_{n}\right)(x)$ converges to $\left(P_{t} f\right)(x)$ uniformly in $[0, T] \times \overline{\Omega^{\prime}}$ for all $T>0$ and all bounded sets $\Omega^{\prime} \subset \Omega$. Finally, $P_{t}$ can be represented in the form

$$
\left(P_{t} f\right)(x)=\int_{\Omega} f(y) p(t, x ; d y), \quad t>0, x \in \bar{\Omega},
$$

where $p(t, x ; d y)$ is a positive finite Borel measure on $\Omega$.

Proof. We may assume that $f=0$. Let $\left(f_{n}\right)_{n \in \mathbb{N}}$ be a bounded sequence in $C_{\mathrm{b}}(\bar{\Omega})$ that converges pointwise to zero in $\Omega$, and set $u_{n}(t, x)=P_{t} f_{n}(x)$. 
Using the local Schauder estimate (3.4) and the maximum principle it follows that the sequence $\left(u_{n}\right)$ is bounded in $C^{1+\alpha / 2,2+\alpha}\left([\varepsilon, T] \times \overline{\Omega^{\prime}}\right)$ for all $0<\varepsilon<T$ and all bounded $\Omega^{\prime} \subset \Omega$. Therefore there exist a subsequence $u_{n_{k}}$ and a function $u \in C^{1,2}(] 0, \infty[\times \bar{\Omega})$ such that $u_{n_{k}}$ converges to $u$ in $C^{1,2}\left([\varepsilon, T] \times \overline{\Omega^{\prime}}\right)$ for all $0<\varepsilon<T$ and for all bounded $\Omega^{\prime} \subset \Omega$. The function $u$ is a bounded solution of the equation

$$
u_{t}-\mathcal{A} u=0 \quad \text { in }(0, \infty) \times \Omega,
$$

and it satisfies the boundary condition

$$
\frac{\partial u}{\partial \nu}=0 \quad \text { in }(0, \infty) \times \partial \Omega .
$$

Now we show that $u$ is continuous up to $t=0$ and that $u(0, x)=0$ in order to conclude that $u \equiv 0$, by Proposition 2.1. Let $\Omega_{0}, \Omega_{1}$ and $\theta$ be as in the proof of Theorem 3.1 and set $v_{n}(t, x)=\theta(x) u_{n}(t, x)$. Then we can write

$$
v_{n}(t)=T(t)\left(\theta f_{n}\right)+\int_{0}^{t} T(t-s) \psi_{n}(s) d s
$$

where $T(t)$ is the semigroup generated by the realization of $\mathcal{A}$ in $C\left(\bar{\Omega}_{0}\right)$ with Neumann boundary condition and

$$
\psi_{n}(t, x)=-u_{n}(t, x)(\mathcal{A}+V(x)) \theta(x)-2 \sum_{i, j=1}^{N} q_{i j}(x) D_{i} u_{n}(t, x) D_{j} \theta(x) .
$$

From the gradient estimate (3.3) and the boundedness of $\left(f_{n_{k}}\right)_{k \in \mathbb{N}}$ it follows that

$$
\left|v_{n_{k}}(t, x)\right| \leq\left|\left(T(t)\left(\theta f_{n_{k}}\right)\right)(x)\right|+C \sqrt{t}, \quad x \in \bar{\Omega}_{0}, 0 \leq t \leq T, k \in \mathbb{N},
$$

where $C>0$ is a constant independent of $k \in \mathbb{N}$. For all $1<p<\infty$ the semigroup $(T(t))$ extends to an analytic semigroup in $L^{p}\left(\Omega_{0}\right)$ (see [14, Section 3.1.1]), and for $p>N$ the domain of the generator of $T(t)$ in $L^{p}\left(\Omega_{0}\right)$ is embedded in $C\left(\bar{\Omega}_{0}\right)$; since $\theta f_{n_{k}}$ converges to zero in $L^{p}\left(\Omega_{0}\right)$ it follows that $T(t)\left(\theta f_{n_{k}}\right)$ converges to zero uniformly in $\Omega_{0}$. Thus letting $k \rightarrow \infty$ in (3.8) we get

$$
|u(t, x)| \leq C \sqrt{t}, \quad 0<t<T, x \in \bar{\Omega}_{1},
$$

which implies that $u$ is continuous at $\left(0, x_{0}\right)$ for all $x_{0} \in \bar{\Omega}_{1}$. Since $\Omega_{1} \subset \Omega$ is arbitrary, we see that $u$ is continuous at $t=0$ with $u(0, x)=0$.

Therefore $u \equiv 0$ and the subsequence $u_{n_{k}}$ converges to zero in $C^{1,2}\left([\varepsilon, T] \times \overline{\Omega^{\prime}}\right)$ for all $0<\varepsilon<T$ and bounded $\Omega^{\prime} \subset \Omega$. As in the proof of Theorem 3.1 one can prove that the whole sequence $\left(u_{n}\right)_{n \in \mathbb{N}}$ converges to zero in $C^{1,2}\left([\varepsilon, T] \times \overline{\Omega^{\prime}}\right)$ for all $0<\varepsilon<T$ and bounded $\Omega^{\prime} \subset \Omega$, as stated. 
Suppose now that $\left(f_{n}\right)_{n \in \mathbb{N}}$ converges to zero uniformly on compact subsets of $\bar{\Omega}$. By (3.8) we have

$$
\left|u_{n}(t, x)\right| \leq\left\|T(t)\left(\theta f_{n}\right)\right\|_{\infty}+C \sqrt{t} \leq\left\|\theta f_{n}\right\|_{\infty}+C \sqrt{t}, \quad x \in \bar{\Omega}_{1}, 0 \leq t \leq T,
$$

where $C>0$ does not depend on $n \in \mathbb{N}$. Therefore for all $\varepsilon>0$ we have

$$
\left\|u_{n}\right\|_{C\left([0, T] \times \bar{\Omega}_{1}\right)} \leq\left\|\theta f_{n}\right\|_{\infty}+C \sqrt{\varepsilon}+\left\|u_{n}\right\|_{C\left([\varepsilon, T] \times \bar{\Omega}_{1}\right)} .
$$

By taking into account the first step of the proof this yields

$$
\limsup _{n \rightarrow \infty}\left\|u_{n}\right\|_{C\left([0, T] \times \bar{\Omega}_{1}\right)} \leq C \sqrt{\varepsilon},
$$

that is, $u_{n}$ converges to zero uniformly in $[0, T] \times \bar{\Omega}_{1}$. Since $\Omega_{1}$ is arbitrary, the conclusion follows.

We can now prove (3.7). By the Riesz representation theorem, for every $x \in \bar{\Omega}$ there exists a positive finite Borel measure $p(t, x ; d y)$ in $\Omega$ such that

$$
\left(P_{t} f\right)(x)=\int_{\Omega} f(y) p(t, x ; d y), \quad f \in C_{0}(\Omega) .
$$

If $f \in C_{\mathrm{b}}(\bar{\Omega})$, we consider a bounded sequence $\left(f_{n}\right)_{n \in \mathbb{N}} \subset C_{0}(\Omega)$ which converges to $f$ uniformly on compact sets of $\Omega$. Writing (3.9) for $f_{n}$ and letting $n \rightarrow \infty$ we obtain the statement for $f \in C_{\mathrm{b}}(\bar{\Omega})$, by dominated convergence.

By a straightforward application of the semigroup law, from Proposition 3.2 it follows that estimate (3.3) extends to the whole half-line $[0, \infty[$.

Corollary 3.3. For all $\omega>0$ there exists $C_{\omega}>0$ such that

$$
\left\|\nabla P_{t} f\right\|_{\infty} \leq C_{\omega} \frac{e^{\omega t}}{\sqrt{t}}\|f\|_{\infty}, \quad t>0, f \in C_{\mathrm{b}}(\bar{\Omega}) .
$$

We remark that the semigroup $\left(P_{t}\right)_{t \geq 0}$ is not strongly continuous in $C_{\mathrm{b}}(\bar{\Omega})$ in general: this is shown by the example $\Omega=\mathbb{R}^{N}$ and $\mathcal{A}=\Delta$. Following the approach in [19], we introduce the weak generator $(\widehat{A}, D(\widehat{A}))$ defined by

$$
\begin{gathered}
D(\widehat{A})=\left\{f \in C_{\mathrm{b}}(\bar{\Omega}): \sup _{t \in(0,1)} \frac{\left\|P_{t} f-f\right\|}{t}<\infty \text { and } \exists g \in C_{\mathrm{b}}(\bar{\Omega})\right. \text { such that } \\
\left.\lim _{t \rightarrow 0} \frac{\left(P_{t} f\right)(x)-f(x)}{t}=g(x), \forall x \in \bar{\Omega}\right\}, \\
\widehat{A} f(x)=\lim _{t \rightarrow 0} \frac{\left(P_{t} f\right)(x)-f(x)}{t}, \quad f \in D(\widehat{A}), x \in \bar{\Omega} .
\end{gathered}
$$

The following results are proved in [19]: if $f \in D(\widehat{A})$, then $P_{t} f \in D(\widehat{A})$ and $\widehat{A} P_{t} f=P_{t} \widehat{A} f$ for all $t \geq 0$. Moreover we have $(0, \infty) \subset \varrho(\widehat{A}),\|R(\lambda, \widehat{A})\|$ 
$\leq 1 / \lambda$ and

$$
(R(\lambda, \widehat{A}) f)(x)=\int_{0}^{\infty} e^{-\lambda t}\left(P_{t} f\right)(x) d t, \quad x \in \bar{\Omega},
$$

and $R(\lambda, \widehat{A})$ is surjective from $C_{\mathrm{b}}(\bar{\Omega})$ onto $D(\widehat{A})$ for all $\lambda>0$. Our aim now is to find a solution of problem (1.2) and to prove that $\widehat{A}$ coincides with the operator $\mathcal{A}$.

Proposition 3.4. For all $f \in C_{\mathrm{b}}(\bar{\Omega})$ and $\lambda>0$, the function $u=$ $R(\lambda, \widehat{A}) f$ belongs to $D(\mathcal{A})$ and solves problem $(1.2)$. Moreover $D(\widehat{A})=D(\mathcal{A})$ and $\widehat{A} v=\mathcal{A} v$ for all $v \in D(\mathcal{A})$.

Proof. Let $f \in C_{\mathrm{b}}(\bar{\Omega})$ and let $u=R(\lambda, \widehat{A}) f$. For all $n \in \mathbb{N}$, let $u_{n}=R_{n}(\lambda, \mathcal{A}) f \in D_{n}(\mathcal{A})$, where $R_{n}(\lambda, \mathcal{A})$ is the resolvent of the operator $\left(\mathcal{A}, D_{n}(\mathcal{A})\right)$, that is,

$$
u_{n}(x)=\int_{0}^{\infty} e^{-\lambda t}\left(T_{n}(t) f\right)(x) d t, \quad x \in \bar{\Omega}_{n} .
$$

Taking into account the contractivity of $T_{n}(t)$, we have

$$
\left\|u_{n}\right\|_{\infty} \leq \frac{1}{\lambda}\|f\|_{\infty}, \quad\left\|\mathcal{A} u_{n}\right\|_{\infty} \leq 2\|f\|_{\infty}
$$

for all $n \in \mathbb{N}$, and then from Theorem 3.1 and by dominated convergence it follows that

$$
\lim _{n \rightarrow \infty} u_{n}=u
$$

pointwise in $\bar{\Omega}$ and in $L^{p}\left(\Omega_{k}\right)$ for all $k \in \mathbb{N}$. Furthermore, by the $L^{p}$ estimates we have

$$
\left\|u_{n}-u_{m}\right\|_{W^{2, p}\left(\Omega_{k}\right)} \leq c(p, k)\left\|u_{n}-u_{m}\right\|_{L^{p}\left(\Omega_{k+1}\right)}, \quad n, m>k,
$$

for all $p \in(1, \infty)$, where $c(p, k)>0$ is a constant. Consequently $u_{n}$ converges to $u$ in $W^{2, p}\left(\Omega_{k}\right)$ for all $k \in \mathbb{N}$. Hence $u \in W^{2, p}\left(\Omega \cap B_{R}\right)$ for all $R<\infty$. Moreover by Sobolev embedding $u_{n}$ converges to $u$ in $C^{1}\left(\bar{\Omega}_{k}\right)$ for all $k \in \mathbb{N}$, and hence we deduce that $\partial u / \partial \nu=0$ in $\partial \Omega$. Finally, letting $n \rightarrow \infty$ in the equation $\lambda u_{n}-\mathcal{A} u_{n}=f$ shows that $\lambda u-\mathcal{A} u=f$ in $\Omega$. Therefore $u$ belongs to $D(\mathcal{A})$ and is a solution of problem $(1.2)$.

In particular, since $R(\lambda, \widehat{A})$ is surjective from $C_{\mathrm{b}}(\bar{\Omega})$ onto $D(\widehat{A})$, it follows that $D(\widehat{A}) \subset D(\mathcal{A})$. Conversely, let $u \in D(\mathcal{A})$ and define $f=\lambda u-\mathcal{A} u \in$ $C_{\mathrm{b}}(\bar{\Omega})$, where $\lambda \geq \lambda_{0}$ (see (2.5)). Then the function $v=R(\lambda, \widehat{A}) f$ is a bounded solution of problem (1.2). By Proposition 2.3 we have $u=v$, and in particular $u \in D(\widehat{A})$.

A consequence of the gradient estimate $(3.10)$ is that $D(\mathcal{A})$ is continuously embedded in $C_{\mathrm{b}}^{1}(\bar{\Omega})$. 
Proposition 3.5. $D(\mathcal{A}) \subseteq C_{\mathrm{b}}^{1}(\bar{\Omega})$. Moreover for all $\omega>0$ there exists a constant $M_{\omega}>0$ such that

$$
\|\nabla u\|_{\infty} \leq M_{\omega}\|u\|_{\infty}^{1 / 2}\|(\mathcal{A}-\omega) u\|_{\infty}^{1 / 2}
$$

for all $u \in D(\mathcal{A})$.

Proof. Let $u \in D(\mathcal{A}), \omega>0$ and $\lambda>0$. Then the function $f=$ $(\lambda+\omega) u-\mathcal{A} u$ belongs to $C_{\mathrm{b}}(\bar{\Omega})$ and

$$
u(x)=(R(\lambda+\omega, \widehat{A}) f)(x)=\int_{0}^{\infty} e^{-(\lambda+\omega) t}\left(P_{t} f\right)(x) d t, \quad x \in \bar{\Omega} .
$$

By using estimate (3.10), we may differentiate under the integral sign to obtain

$$
\nabla u(x)=\int_{0}^{\infty} e^{-(\lambda+\omega) t}\left(\nabla P_{t} f\right)(x) d t, \quad x \in \Omega,
$$

and

$$
|\nabla u(x)| \leq C_{\omega} \int_{0}^{\infty} \frac{e^{-\lambda t}}{\sqrt{t}} d t\|f\|_{\infty}=\frac{M_{\omega}}{\sqrt{\lambda}}\|f\|_{\infty}, \quad x \in \Omega,
$$

where $M_{\omega}>0$ is a constant. Therefore

$$
\|\nabla u\|_{\infty} \leq M_{\omega}\left(\sqrt{\lambda}\|u\|_{\infty}+\frac{\|(\mathcal{A}-\omega) u\|_{\infty}}{\sqrt{\lambda}}\right),
$$

and, if we take the minimum over $\lambda,(3.14)$ follows.

With the same technique as in Proposition 2.5 we get the following gradient estimate.

Proposition 3.6. For every $T>0$ there exists $C_{T}>0$ such that

$$
\left\|\nabla P_{t} f\right\|_{\infty} \leq C_{T}\left(\|f\|_{\infty}+\|\nabla f\|_{\infty}\right), \quad 0<t \leq T,
$$

for every $f \in C_{\nu}^{1}(\bar{\Omega})$ (see $\left.(1.5)\right)$.

Proof. We may suppose that $V \geq 1$; the general case follows by considering the operator $\mathcal{A}^{\prime}=\mathcal{A}-I$. We give the proof in several steps; first we prove that there exists a constant $C_{T}>0$ such that

$$
\left|\nabla T_{n}(t) f(x)\right| \leq C_{T}\left(\|f\|_{\infty}+\|\nabla f\|_{\infty}\right), \quad 0<t \leq T, x \in \bar{\Omega}_{n},
$$

for every $n \in \mathbb{N}$ and $f \in C_{\nu}^{1}\left(\bar{\Omega}_{n}\right)$. Since $D_{n}(\mathcal{A})$ (see (3.1)) is dense in $C_{\nu}^{1}\left(\bar{\Omega}_{n}\right)$, it is enough to prove $(3.16)$ for $f \in D_{n}(\mathcal{A})$.

Let $f \in D_{n}(\mathcal{A})$ and define

$$
w(t, x)=u^{2}(t, x)+a|\nabla u(t, x)|^{2}, \quad t>0, x \in \Omega_{n},
$$


where $u(t, x)=\left(T_{n}(t) f\right)(x)$ and $a>0$ is a constant. Then $w \in C\left([0, T] \times \bar{\Omega}_{n}\right)$ $\left.\left.\left.\left.\cap C^{0,1}(] 0, T\right] \times \bar{\Omega}_{n}\right) \cap C^{1,2}(] 0, T\right] \times \Omega_{n}\right)$ and from Lemma 2.4 it follows that

$$
\frac{\partial w}{\partial \nu}(t, x) \leq 0, \quad t>0, x \in \partial \Omega_{n}
$$

Moreover $w$ satisfies the equation

$$
w_{t}(t, x)-\mathcal{A} w(t, x)=-2 \sum_{i, j=1}^{N} q_{i j}(x) D_{i} u(t, x) D_{j} u(t, x)+h_{1}(t, x)+h_{2}(t, x),
$$

where

$$
\begin{aligned}
h_{1}(t, x)= & 2 a \sum_{i, j=1}^{N} D_{i} F_{j}(x) D_{i} u(t, x) D_{j} u(t, x)-a V(x)|\nabla u(t, x)|^{2} \\
& -2 a u(t, x) \nabla u(t, x) \cdot \nabla V(x)-V(x) u^{2}(t, x), \\
h_{2}(t, x)= & 2 a\left(\sum_{i, j, k=1}^{N} D_{k} q_{i j}(x) D_{k} u(t, x) D_{i j} u(t, x)\right. \\
& \left.-\sum_{i, j, k=1}^{N} q_{i j}(x) D_{i k} u(t, x) D_{j k} u(t, x)\right) .
\end{aligned}
$$

The same estimates from the proof of Proposition 2.5 show that there exists a value of $a>0$ independent of $n$ such that

$$
w_{t}(t, x)-\mathcal{A} w(t, x) \leq 0, \quad 0<t \leq T, x \in \Omega_{n} .
$$

Therefore the classical maximum principle yields

$$
w(t, x) \leq \sup _{x \in \bar{\Omega}_{n}} w(0, x) \leq\|f\|_{\infty}^{2}+a\|\nabla f\|_{\infty}^{2}, \quad 0<t \leq T, x \in \bar{\Omega}_{n},
$$

which implies (3.16) with $C_{T}=a^{-1 / 2} \vee 1$.

Let now $f \in C_{\nu}^{1}(\bar{\Omega})$. For all $k \in \mathbb{N}$, let $\theta_{k} \in C_{\mathrm{b}}^{1}(\bar{\Omega})$ be a function with bounded support such that

$$
0 \leq \theta_{k} \leq 1, \quad\left\|\nabla \theta_{k}\right\|_{\infty} \leq L, \quad \theta_{k}=1 \quad \text { in } \Omega_{k}, \quad \frac{\partial \theta_{k}}{\partial \nu}=0 \quad \text { in } \partial \Omega,
$$

where $L>0$ is a constant independent of $k \in \mathbb{N}$, and set $f_{k}=\theta_{k} f$. Then for all $n \in \mathbb{N}$ such that $\operatorname{supp}\left(\theta_{k}\right) \subset \Omega_{n}$ we have

$$
\frac{\partial f_{k}}{\partial \nu}(x)=\frac{\partial \theta_{k}}{\partial \nu}(x) f(x)+\theta_{k}(x) \frac{\partial f}{\partial \nu}(x)=0, \quad x \in \partial \Omega_{n},
$$

that is, $f_{k} \in C_{\nu}^{1}\left(\bar{\Omega}_{n}\right)$. Then $T_{n}(t) f_{k}$ satisfies estimate (3.16), and letting $n \rightarrow \infty$ we get

$$
\begin{aligned}
\left|\nabla P_{t} f_{k}(x)\right| & \leq C_{T}\left(\left\|f_{k}\right\|_{\infty}+\left\|\nabla f_{k}\right\|_{\infty}\right) \\
& \leq C_{T}\left((1+L)\|f\|_{\infty}+\|\nabla f\|_{\infty}\right), \quad 0<t \leq T, x \in \bar{\Omega} .
\end{aligned}
$$


Taking into account Proposition 3.2 and letting $k \rightarrow \infty$ yields the statement.

As a consequence we get the following result which will be used in what follows.

Proposition 3.7. If $f \in C_{\nu}^{1}(\bar{\Omega})$ then the function $\nabla P_{t} f$ is continuous in $[0, \infty) \times \bar{\Omega}$.

Proof. Let $f \in C_{\nu}^{1}(\bar{\Omega})$. Taking account of Theorem 3.1 we only have to prove that $\nabla P_{t} f$ is continuous at $t=0$. Let $x_{0} \in \bar{\Omega}$ be fixed and $\Omega_{0}, \Omega_{1}, \theta$ and $T(t)$ be as in the proof of Theorem 3.1. We set

$$
v(t, x)=\theta(x)\left(P_{t} f\right)(x), \quad t \geq 0, x \in \bar{\Omega}_{0},
$$

and we prove that $\nabla v$ is continuous at $\left(0, x_{0}\right)$; since $v(t, x)=\left(P_{t} f\right)(x)$ for all $x \in \Omega_{1}$, the conclusion follows. We can write

$$
v(t)=T(t)(\theta f)+\int_{0}^{t} T(t-s) \psi(s) d s
$$

where

$$
\psi(t, x)=-P_{t} f(x)(\mathcal{A}+V(x)) \theta(x)-2 \sum_{i, j=1}^{N} q_{i j}(x) D_{i} P_{t} f(x) D_{j} \theta(x) .
$$

From Proposition 3.6 it follows that

$$
\|\psi(t)\|_{\infty} \leq C_{T}\left(\|f\|_{\infty}+\|\nabla f\|_{\infty}\right), \quad 0<t \leq T
$$

for some $C_{T}>0$, for all fixed $T>0$, and then by (2.12) we have

$$
\|\nabla T(t-s) \psi(s)\|_{\infty} \leq \frac{C}{\sqrt{t-s}}\left(\|f\|_{\infty}+\|\nabla f\|_{\infty}\right), \quad 0<s<t \leq T,
$$

for some $C>0$. Therefore

$$
\begin{aligned}
\left|\nabla v(t, x)-\nabla f\left(x_{0}\right)\right| \leq & \left|\nabla T(t)(\theta f)(x)-\nabla f\left(x_{0}\right)\right| \\
& +2 C \sqrt{t}\left(\|f\|_{\infty}+\|\nabla f\|_{\infty}\right), \quad 0<t \leq T, x \in \bar{\Omega}_{0} .
\end{aligned}
$$

Taking account of

$$
\lim _{(t, x) \rightarrow\left(0, x_{0}\right)}\left|\nabla T(t)(\theta f)(x)-\nabla f\left(x_{0}\right)\right|=0,
$$

we conclude that $\nabla v$ is continuous at $\left(0, x_{0}\right)$. Relation (3.17) is immediate if $\theta f \in D_{\nu}(\mathcal{A})$, where $D_{\nu}(\mathcal{A})$ is the domain of the generator of $T(t)$, as in (2.10). Indeed, in this case $T(t)(\theta f)$ belongs to $C\left([0, \infty) ; D_{\nu}(\mathcal{A})\right)$ and $D_{\nu}(\mathcal{A}) \subset C_{\nu}^{1}\left(\bar{\Omega}_{0}\right)$. In general we have $\theta f \in C_{\nu}^{1}\left(\bar{\Omega}_{0}\right)$ (see $\left.(3.5)\right)$, and $(3.17)$ follows by approximation, since $D_{\nu}(\mathcal{A})$ is dense in $C_{\nu}^{1}\left(\bar{\Omega}_{0}\right)$.

REMARK 3.8. In the case $\Omega=\mathbb{R}^{N}$ the compactness of $P_{t}$ in $C_{\mathrm{b}}\left(\mathbb{R}^{N}\right)$ has been studied in [16]. The results extend to the case $\Omega \neq \mathbb{R}^{N}$, with the same proofs adapted to the Neumann problem. Assume that $V \equiv 0$, i.e. consider 
the conservative case where $P_{t} \mathbb{1}=\mathbb{1}$. First, $P_{t}$ is compact in $C_{\mathrm{b}}(\bar{\Omega})$ for all $t>0$ if and only if for all $t, \varepsilon>0$ there exists a bounded set $\Omega^{\prime} \subset \Omega$ such that $p\left(t, x, \Omega^{\prime}\right) \geq 1-\varepsilon$ for all $x \in \bar{\Omega}$. Secondly, if there exists a positive function $\psi \in C^{2}$ such that

$$
\lim _{|x| \rightarrow \infty} \psi(x)=\infty, \quad \frac{\partial \psi}{\partial \nu}(x)=0, \quad x \in \partial \Omega, \quad \mathcal{A} \psi(x) \leq-g(\psi(x)), \quad x \in \Omega,
$$

where $g:\left[0, \infty\left[\rightarrow \mathbb{R}\right.\right.$ is a convex function such that $\lim _{x \rightarrow \infty} g(x)=\infty$ and $1 / g$ is integrable at $\infty$, then $P_{t}$ is compact in $C_{\mathrm{b}}(\bar{\Omega})$ for all $t>0$.

4. Pointwise gradient estimates. In the whole section we assume that $V \equiv 0$, which implies that $P_{t} \mathbb{1}=\mathbb{1}$ for all $t>0$ thanks to uniqueness. Actually this is a necessary condition for the estimates that we are going to prove. Indeed, taking $f=\mathbb{1}$ in (4.1) shows that $P_{t} \mathbb{1}=\mathbb{1}$.

Proposition 4.1. Suppose $q_{i j}(x) \equiv \delta_{i j}$ for all $i, j=1, \ldots, N$. Then for every $p \geq 1$ and $f \in C_{\nu}^{1}(\bar{\Omega})$ we have

$$
\left|\nabla P_{t} f(x)\right|^{p} \leq e^{p k_{0} t} P_{t}\left(|\nabla f|^{p}\right)(x), \quad t \geq 0, x \in \bar{\Omega} .
$$

Proof. It is sufficient to prove the case $p=1$. For $p>1$, we observe that since $P_{t} \mathbb{1}=\mathbb{1}$ the measures $p(t, x ; d y)$ given by Proposition 3.2 are probability measures, and then Jensen's inequality yields

$$
\left|\nabla P_{t} f(x)\right|^{p} \leq\left(e^{k_{0} t} P_{t}(|\nabla f|)(x)\right)^{p} \leq e^{k_{0} p t} P_{t}\left(|\nabla f|^{p}\right)(x) .
$$

Let $f \in C_{\nu}^{1}(\bar{\Omega})$ and let $\varepsilon>0$ be fixed. Set $u(t, x)=P_{t} f(x)$ and define the function

$$
w(t, x)=\left(|\nabla u(t, x)|^{2}+\varepsilon\right)^{1 / 2}, \quad t>0, x \in \Omega .
$$

From Propositions 3.6 and 3.7 it follows that $w$ is bounded and continuous in $\left[0, \infty\left[\times \bar{\Omega}\right.\right.$. Since $u \in C_{\mathrm{loc}}^{1+\alpha / 2,2+\alpha}(] 0, \infty[\times \bar{\Omega})$ (see Theorem 3.1), we find that $w \in C^{0,1}(] 0, \infty[\times \bar{\Omega})$. Finally, from [12, Theorem 8.12.1] we deduce that $w \in C^{1,2}(] 0, \infty[\times \Omega)$. From Lemma 2.4 it follows that

$$
\frac{\partial w}{\partial \nu}(t, x)=\frac{1}{2}\left(|\nabla u(t, x)|^{2}+\varepsilon\right)^{-1 / 2} \frac{\partial}{\partial \nu}|\nabla u|^{2}(t, x) \leq 0, \quad t>0, x \in \partial \Omega .
$$

A straightforward computation shows that $w$ satisfies the equation

$$
w_{t}(t, x)-\mathcal{A} w(t, x)=g_{1}(t, x)+g_{2}(t, x)
$$

where

$$
\begin{aligned}
& g_{1}=\left(|\nabla u|^{2}+\varepsilon\right)^{-1 / 2} \sum_{i, j=1}^{N}\left(D_{i} F_{j}\right)\left(D_{i} u\right)\left(D_{j} u\right) \\
& g_{2}=\left(|\nabla u|^{2}+\varepsilon\right)^{-3 / 2} \sum_{i=1}^{N}\left(\sum_{j=1}^{N}\left(D_{j} u\right)\left(D_{i j} u\right)\right)^{2}-\left(|\nabla u|^{2}+\varepsilon\right)^{-1 / 2} \sum_{i, j=1}^{N}\left(D_{i j} u\right)^{2} .
\end{aligned}
$$


We now estimate the functions $g_{1}$ and $g_{2}$. Since

$$
\begin{aligned}
\left(|\nabla u|^{2}+\varepsilon\right)^{-3 / 2} \sum_{i=1}^{N}\left(\sum_{j=1}^{N} D_{j} u D_{i j} u\right)^{2} & \leq\left(|\nabla u|^{2}+\varepsilon\right)^{-3 / 2}|\nabla u|^{2} \sum_{i, j=1}^{N}\left(D_{i j} u\right)^{2} \\
& \leq\left(|\nabla u|^{2}+\varepsilon\right)^{-1 / 2} \sum_{i, j=1}^{N}\left(D_{i j} u\right)^{2},
\end{aligned}
$$

it follows that $g_{2} \leq 0$. On the other hand using (2.3) we obtain

$$
\begin{aligned}
g_{1}(t, x) & \leq k_{0}\left(|\nabla u(t, x)|^{2}+\varepsilon\right)^{-1 / 2}|\nabla u(t, x)|^{2} \\
& =k_{0} w-k_{0} \varepsilon\left(|\nabla u(t, x)|^{2}+\varepsilon\right)^{-1 / 2} .
\end{aligned}
$$

If $k_{0} \geq 0$ we immediately have

$$
g_{1}(t, x) \leq k_{0} w
$$

whereas if $k_{0}<0$, we have

$$
g_{1}(t, x) \leq k_{0} w-k_{0} \sqrt{\varepsilon} .
$$

In any case we obtain

$$
w_{t}-\mathcal{A} w \leq k_{0}\left(w-\delta_{\varepsilon}\right) \quad \text { where } \quad \delta_{\varepsilon}= \begin{cases}0 & k_{0} \geq 0 \\ \sqrt{\varepsilon} & k_{0}<0\end{cases}
$$

Therefore the function $v=w-\delta_{\varepsilon}$ satisfies

$$
\begin{cases}v_{t}(t, x)-\mathcal{A} v(t, x) \leq k_{0} v(t, x), & t>0, x \in \Omega, \\ \frac{\partial v}{\partial \nu}(t, x) \leq 0, & t>0, x \in \partial \Omega, \\ v(0, x)=\left(|\nabla f(x)|^{2}+\varepsilon\right)^{1 / 2}-\delta_{\varepsilon}, & x \in \bar{\Omega} .\end{cases}
$$

On the other hand, the function

$$
z(t, x)=e^{k_{0} t} P_{t}\left(\left(|\nabla f|^{2}+\varepsilon\right)^{1 / 2}\right)(x), \quad t>0, x \in \Omega,
$$

solves the problem

$$
\begin{cases}z_{t}(t, x)-\mathcal{A} z(t, x)=k_{0} z(t, x), & t>0, x \in \Omega, \\ \frac{\partial z}{\partial \nu}(t, x)=0, & t>0, x \in \partial \Omega, \\ z(0, x)=\left(|\nabla f(x)|^{2}+\varepsilon\right)^{1 / 2}, & x \in \bar{\Omega} .\end{cases}
$$

Therefore Proposition 2.1 applied to $v-z$ and to the operator $\mathcal{A}+k_{0} I$ yields $v \leq z$, that is,

$$
\left(|\nabla u(t, x)|^{2}+\varepsilon\right)^{1 / 2}-\delta_{\varepsilon} \leq e^{k_{0} t} P_{t}\left(\left(|\nabla f|^{2}+\varepsilon\right)^{1 / 2}\right)(x), \quad t \geq 0, x \in \bar{\Omega} .
$$

Letting $\varepsilon \rightarrow 0$ yields estimate (4.1) with $p=1$. 
We now consider the case of variable second order coefficients. Under the assumption

$$
\sum_{i, j=1}^{N}\left(\nabla q_{i j}(x) \cdot \xi\right)^{2} \leq q_{0} \mu(x)|\xi|^{2}, \quad x \in \Omega, \xi \in \mathbb{R}^{N},
$$

which is slightly stronger than (2.2), we generalize the previous result when $p>1$.

Proposition 4.2. Suppose that (4.2) holds. Then

$$
\left|\nabla P_{t} f(x)\right|^{p} \leq e^{\sigma_{p} t} P_{t}\left(|\nabla f|^{p}\right)(x), \quad t \geq 0, x \in \bar{\Omega},
$$

for all $p>1$ and $f \in C_{\nu}^{1}(\bar{\Omega})$, where

$$
\sigma_{p}= \begin{cases}p k_{0}+\frac{p}{4} q_{0} & \text { if } p \geq 2, \\ p k_{0}+\frac{p}{4(p-1)} q_{0} & \text { if } 1<p<2 .\end{cases}
$$

Proof. Let $f \in C_{\nu}^{1}(\bar{\Omega})$ be fixed. We first prove the statement for $p=2$. Consider the function

$$
w(t, x)=|\nabla u(t, x)|^{2}, \quad t>0, x \in \Omega,
$$

where $u(t, x)=\left(P_{t} f\right)(x)$; then $w \in C\left(\left[0, \infty[\times \bar{\Omega}) \cap C^{0,1}(] 0, \infty[\times \bar{\Omega}) \cap\right.\right.$ $C^{1,2}(] 0, \infty[\times \Omega)$, and from Lemma 2.4 we have

$$
\frac{\partial w}{\partial \nu}(t, x) \leq 0, \quad t>0, x \in \partial \Omega
$$

Moreover it is readily seen that

$$
w_{t}(t, x)-\mathcal{A} w(t, x)=f_{0}(t, x),
$$

where

$$
f_{0}=2\left(\sum_{i, j, k} D_{k} q_{i j} D_{k} u D_{i j} u+\sum_{j, k} D_{k} F_{j} D_{k} u D_{j} u-\sum_{i, j, k} q_{i j} D_{i k} u D_{j k} u\right) .
$$

From (4.2) it follows that

$$
\begin{aligned}
\sum_{i, j, k=1}^{N} D_{k} q_{i j}(x) D_{k} u D_{i j} u & \leq\left(\sum_{i, j=1}^{N}\left(D_{i j} u\right)^{2}\right)^{1 / 2}\left(\sum_{i, j=1}^{N}\left(\nabla q_{i j} \cdot \nabla u\right)^{2}\right)^{1 / 2} \\
& \leq\left(\sum_{i, j=1}^{N}\left(D_{i j} u\right)^{2}\right)^{1 / 2}\left(q_{0} \mu(x)|\nabla u|^{2}\right)^{1 / 2} \\
& \leq \mu(x) \sum_{i, j=1}^{N}\left(D_{i j} u\right)^{2}+\frac{1}{4} q_{0}|\nabla u|^{2}
\end{aligned}
$$


and then using (2.3) we get

$$
\begin{aligned}
f_{0}(t, x) & \leq 2\left(\mu(x) \sum_{i, j=1}^{N}\left(D_{i j} u\right)^{2}+\frac{1}{4} q_{0}|\nabla u|^{2}+k_{0}|\nabla u|^{2}-\mu(x) \sum_{i, j=1}^{N}\left(D_{i j} u\right)^{2}\right) \\
& =\left(2 k_{0}+q_{0} / 2\right)|\nabla u|^{2}=\sigma_{2}|\nabla u|^{2} .
\end{aligned}
$$

On the other hand the function

$$
z(t, x)=e^{\sigma_{2} t} P_{t}\left(|\nabla f|^{2}\right)(x), \quad t>0, x \in \Omega,
$$

is the solution of the problem

$$
\begin{cases}z_{t}(t, x)-\mathcal{A} z(t, x)=\sigma_{2} z(t, x), & t>0, x \in \Omega, \\ \frac{\partial z}{\partial \nu}(t, x)=0, & t>0, x \in \partial \Omega \\ z(0, x)=|\nabla f(x)|^{2}, & x \in \bar{\Omega} .\end{cases}
$$

Using Proposition 2.1 we can conclude that $w \leq z$, which is $(4.3)$ with $p=2$.

Now the case $p>2$ follows easily by applying Jensen's inequality:

$$
\left|\nabla P_{t} f(x)\right|^{p} \leq\left(e^{\sigma_{2} t} P_{t}\left(|\nabla f|^{2}\right)(x)\right)^{p / 2} \leq e^{\sigma_{p} t} P_{t}\left(|\nabla f|^{p}\right)(x), \quad t>0, x \in \Omega .
$$

Assume $1<p<2$. Fix $\varepsilon>0$ and define the function

$$
w(t, x)=\left(|\nabla u(t, x)|^{2}+\varepsilon\right)^{p / 2},
$$

where $u(t, x)=\left(P_{t} f\right)(x)$. Then $w \in C\left(\left[0, \infty[\times \bar{\Omega}) \cap C^{0,1}(] 0, \infty[\times \bar{\Omega}) \cap\right.\right.$ $C^{1,2}(] 0, \infty[\times \Omega)$, and from Lemma 2.4 we have

$$
\frac{\partial w}{\partial \nu}(t, x)=\frac{p}{2}\left(|\nabla u(t, x)|^{2}+\varepsilon\right)^{p / 2-1} \frac{\partial}{\partial \nu}|\nabla u(t, x)|^{2} \leq 0, \quad t>0, x \in \partial \Omega .
$$

Moreover it turns out that

$$
w_{t}(t, x)-\mathcal{A} w(t, x)=f_{1}(t, x)+f_{2}(t, x),
$$

where

$$
\begin{aligned}
& f_{1}=p\left(|\nabla u|^{2}+\varepsilon\right)^{(p-2) / 2} f_{0}, \\
& f_{2}=p(2-p)\left(|\nabla u|^{2}+\varepsilon\right)^{(p-4) / 2} \sum_{i, j, k, h} q_{i j} D_{k} u D_{j k} u D_{h} u D_{i h} u .
\end{aligned}
$$

Taking into account (4.4) for all $\delta>0$ we have

$$
\begin{aligned}
f_{1} \leq & p\left(|\nabla u|^{2}+\varepsilon\right)^{(p-2) / 2}\left(\delta \mu(x) \sum_{i, j=1}^{N}\left(D_{i j} u\right)^{2}\right. \\
& \left.+\frac{1}{4 \delta} q_{0}|\nabla u|^{2}+k_{0}|\nabla u|^{2}-\sum_{i, j, k=1}^{N} q_{i j} D_{j k} u D_{i k} u\right) .
\end{aligned}
$$

As far as $f_{2}$ is concerned, we set $A_{k h}=\sum_{i, j=1}^{N} q_{i j} D_{j k} u D_{i h} u$ and we observe that, since the matrix $A=\left(A_{k h}\right)$ is symmetric and nonnegative definite, 
we have $\sum_{k, h=1}^{N} A_{k h} D_{h} u D_{k} u \leq \operatorname{Tr}(A)|\nabla u|^{2}$, where $\operatorname{Tr}(A)$ denotes the trace of $A$. Therefore

$$
\begin{aligned}
f_{2} & =p(2-p)\left(|\nabla u|^{2}+\varepsilon\right)^{(p-4) / 2} \sum_{k, h=1}^{N} A_{k h} D_{k} u D_{h} u \\
& \leq p(2-p)\left(|\nabla u|^{2}+\varepsilon\right)^{(p-2) / 2} \sum_{i, j, k=1}^{N} q_{i j} D_{j k} u D_{i k} u .
\end{aligned}
$$

Choosing $\delta=p-1$ we get

$$
\begin{aligned}
f_{1}+f_{2} \leq & p\left(|\nabla u|^{2}+\varepsilon\right)^{(p-2) / 2}\left((p-1) \mu(x) \sum_{i, j=1}^{N}\left(D_{i j} u\right)^{2}\right. \\
& \left.+\left(\frac{q_{0}}{4(p-1)}+k_{0}\right)|\nabla u|^{2}+(1-p) \sum_{i, j, k=1}^{N} q_{i j} D_{j k} u D_{i k} u\right) \\
\leq & \left(p k_{0}+\frac{p}{4(p-1)} q_{0}\right)\left(|\nabla u|^{2}+\varepsilon\right)^{(p-2) / 2}|\nabla u|^{2} \\
= & \sigma_{p} w-\varepsilon \sigma_{p}\left(|\nabla u|^{2}+\varepsilon\right)^{(p-2) / 2},
\end{aligned}
$$

which implies

$$
w_{t}-\mathcal{A} w \leq \sigma_{p}\left(w-\delta_{\varepsilon}\right)
$$

where

$$
\delta_{\varepsilon}= \begin{cases}0 & \text { if } \sigma_{p} \geq 0 \\ \varepsilon^{p / 2} & \text { if } \sigma_{p}<0 .\end{cases}
$$

Now the conclusion of the proof is the same as in Proposition 4.1: applying Proposition 2.1 to compare with $z(t, x)=e^{\sigma_{p} t} P_{t}\left(\left(|\nabla f|^{2}+\varepsilon\right)^{p / 2}\right)$ we deduce that

$$
\left(|\nabla u(t, x)|^{2}+\varepsilon\right)^{p / 2}-\delta_{\varepsilon} \leq e^{\sigma_{p} t} P_{t}\left(\left(|\nabla f|^{2}+\varepsilon\right)^{p / 2}\right)(x), \quad t \geq 0, x \in \bar{\Omega},
$$

and then (4.3) follows by letting $\varepsilon \rightarrow 0$.

In the following proposition we deduce from (4.3) another type of pointwise gradient estimate. The basic idea of the proof is taken from [3] where the case $p=2$ is considered.

Proposition 4.3. Assume that (4.2) holds. Then for all $f \in C_{\mathrm{b}}(\bar{\Omega})$ we have

$$
\left|\nabla P_{t} f(x)\right|^{p} \leq\left(\frac{\sigma_{2} \mu_{0}^{-1}}{2\left(1-e^{-\sigma_{2} t}\right)}\right)^{p / 2} P_{t}\left(|f|^{p}\right)(x), \quad t>0, x \in \bar{\Omega},
$$

for all $p \geq 2$, and

$$
\left|\nabla P_{t} f(x)\right|^{p} \leq \frac{c_{p} \mu_{0}^{-1} \sigma_{p}}{t^{p / 2-1}\left(1-e^{-\sigma_{p} t}\right)} P_{t}\left(|f|^{p}\right)(x), \quad t>0, x \in \bar{\Omega},
$$


for all $1<p<2$, where $c_{p}=2^{p} /(p(p-1))^{p / 2}$ and $\sigma_{p}$ is given by Proposition 4.2. When $\sigma_{p}=0$ in (4.5) and (4.6), we replace $\sigma_{p} /\left(1-e^{-\sigma_{p} t}\right)$ by $1 / t$.

Proof. We prove that $T_{n}(t) f$ satisfies estimates (4.5) and (4.6) for $x \in \bar{\Omega}_{n}$, for all $n \in \mathbb{N}$; then the conclusion follows by letting $n \rightarrow \infty$. Fix $n \in \mathbb{N}$ and set $T_{t}=T_{n}(t)$, for simplicity. Note that $T_{t}$ satisfies estimate (4.3) for all the functions in $C_{\nu}^{1}\left(\bar{\Omega}_{n}\right)$.

First we consider the case $p=2$. Let $f \in C_{\mathrm{b}}(\bar{\Omega})$, fix $t>0$ and set

$$
\Phi(s)=T_{s}\left(\left(T_{t-s} f\right)^{2}\right), \quad 0 \leq s \leq t-\varepsilon,
$$

where $\varepsilon>0$. From the analyticity of $T_{t}$ it follows that $g=T_{t-s} f \in D_{n}(\mathcal{A})$ for all $0 \leq s \leq t-\varepsilon$ (we recall that $D_{n}(\mathcal{A})$ is the domain of the generator of $T_{t}$, defined in (3.1)). Moreover from a direct calculation it is readily seen that $g^{2} \in D_{n}(\mathcal{A})$ and

$$
\Phi^{\prime}(s)=\mathcal{A} T_{s}\left(g^{2}\right)-2 T_{s}(g \mathcal{A} g)=T_{s}\left(\mathcal{A}\left(g^{2}\right)-2 g \mathcal{A} g\right)=2 T_{s}(\mathcal{Q}[\nabla g]),
$$

where $\mathcal{Q}$ is defined in (1.10). Thus

$$
\Phi(t-\varepsilon)-\Phi(0)=T_{t-\varepsilon}\left(\left(T_{\varepsilon} f\right)^{2}\right)-\left(T_{t} f\right)^{2}=2 \int_{0}^{t-\varepsilon} T_{s}\left(\mathcal{Q}\left[\nabla T_{t-s} f\right]\right) d s .
$$

Now, applying Proposition 4.2 to $T_{t-s} f$ we obtain

$$
T_{s}\left(\mathcal{Q}\left[\nabla T_{t-s} f\right]\right) \geq \mu_{0} T_{s}\left(\left|\nabla T_{t-s} f\right|^{2}\right) \geq \mu_{0} e^{-\sigma_{2} s}\left|\nabla T_{t} f\right|^{2},
$$

so that

$$
\begin{aligned}
T_{t-\varepsilon}\left(\left(T_{\varepsilon} f\right)^{2}\right)-\left(T_{t} f\right)^{2} & \geq 2 \mu_{0}\left|\nabla T_{t} f\right|^{2} \int_{0}^{t-\varepsilon} e^{-\sigma_{2} s} d s \\
& =\frac{2 \mu_{0}\left(1-e^{-\sigma_{2}(t-\varepsilon)}\right)}{\sigma_{2}}\left|\nabla T_{t} f\right|^{2},
\end{aligned}
$$

and then

$$
\begin{aligned}
\left|\nabla T_{t} f\right|^{2} & \leq \frac{\sigma_{2} \mu_{0}^{-1}}{2\left(1-e^{-\sigma_{2}(t-\varepsilon)}\right)}\left(T_{t-\varepsilon}\left(\left(T_{\varepsilon} f\right)^{2}\right)-\left(T_{t} f\right)^{2}\right) \\
& \leq \frac{\sigma_{2} \mu_{0}^{-1}}{2\left(1-e^{-\sigma_{2}(t-\varepsilon)}\right)} T_{t-\varepsilon}\left(\left(T_{\varepsilon} f\right)^{2}\right) .
\end{aligned}
$$

Letting $\varepsilon \rightarrow 0$ we obtain our claim.

If $p>2$, using Jensen's inequality we get

$$
\left|\nabla T_{t} f\right|^{p} \leq\left(\frac{\sigma_{2} \mu_{0}^{-1}}{2\left(1-e^{-\sigma_{2} t}\right)} T_{t}\left(f^{2}\right)\right)^{p / 2} \leq\left(\frac{\sigma_{2} \mu_{0}^{-1}}{2\left(1-e^{-\sigma_{2} t}\right)}\right)^{p / 2} T_{t}\left(|f|^{p}\right) .
$$

Now assume $1<p<2$. Let first $f \in C_{\mathrm{b}}(\bar{\Omega})$ with $f \geq \delta$ for some $\delta>0$. Fix $t, \varepsilon>0$ and define the function

$$
\Psi(s)=T_{s}\left(\left(T_{t-s} f\right)^{p}\right), \quad 0 \leq s \leq t-\varepsilon .
$$


Then $g=T_{t-s} f \geq \delta>0$ and a straightforward computation shows that

$$
\mathcal{A}\left(g^{p}\right)=p g^{p-1} \mathcal{A} g+p(p-1) g^{p-2} \mathcal{Q}[\nabla g], \quad \frac{\partial g^{p}}{\partial \nu}=p g^{p-1} \frac{\partial g}{\partial \nu},
$$

which implies that $g^{p} \in D_{n}(\mathcal{A})$, since $g \in D_{n}(\mathcal{A})$. Moreover

$$
\Psi^{\prime}(s)=T_{s}\left(\mathcal{A}\left(g^{p}\right)-p g^{p-1} \mathcal{A} g\right)=p(p-1) T_{s}\left(\left(T_{t-s} f\right)^{p-2} \mathcal{Q}\left[\nabla T_{t-s} f\right]\right),
$$

and hence

$$
T_{t-\varepsilon}\left(\left(T_{\varepsilon} f\right)^{p}\right)-\left(T_{t} f\right)^{p}=p(p-1) \int_{0}^{t-\varepsilon} T_{s}\left(\left(T_{t-s} f\right)^{p-2} \mathcal{Q}\left[\nabla T_{t-s} f\right]\right) d s .
$$

Applying Proposition 4.2 and Hölder's inequality we get, for all $\beta \in \mathbb{R}$,

$\left|\nabla T_{t} f\right|^{p}=\left|\nabla T_{s} T_{t-s} f\right|^{p} \leq e^{\sigma_{p} s} T_{s}\left(\left|\nabla T_{t-s} f\right|^{p}\right)$

$$
\begin{aligned}
& =e^{\sigma_{p} s} T_{s}\left(\left|\nabla T_{t-s} f\right|^{p}\left(T_{t-s} f\right)^{-\beta}\left(T_{t-s} f\right)^{\beta}\right) \\
& \leq \mu_{0}^{-1} e^{\sigma_{p} s}\left\{T_{s}\left(\mathcal{Q}\left[\nabla T_{t-s} f\right]\left(T_{t-s} f\right)^{-2 \beta / p}\right)\right\}^{p / 2}\left\{T_{s}\left(T_{t-s} f\right)^{2 \beta /(2-p)}\right\}^{1-p / 2} .
\end{aligned}
$$

Choosing $\beta=p(2-p) / 2$ and using Jensen's and Young's inequalities we get, for all $\eta>0$,

$$
\begin{aligned}
\left|\nabla T_{t} f\right|^{p} \leq & \mu_{0}^{-1} e^{\sigma_{p} s}\left\{T_{s}\left(\mathcal{Q}\left[\nabla T_{t-s} f\right]\left(T_{t-s} f\right)^{p-2}\right)\right\}^{p / 2}\left\{T_{s}\left(T_{t-s} f\right)^{p}\right\}^{1-p / 2} \\
\leq & \mu_{0}^{-1} e^{\sigma_{p} s}\left\{T_{s}\left(\mathcal{Q}\left[\nabla T_{t-s} f\right]\left(T_{t-s} f\right)^{p-2}\right)\right\}^{p / 2}\left\{T_{t}\left(f^{p}\right)\right\}^{1-p / 2} \\
\leq & \mu_{0}^{-1} e^{\sigma_{p} s}\left\{\frac{p}{2} \eta^{2 / p} T_{s}\left(\mathcal{Q}\left[\nabla T_{t-s} f\right]\left(T_{t-s} f\right)^{p-2}\right)\right. \\
& \left.+\left(1-\frac{p}{2}\right) \eta^{2 /(p-2)} T_{t}\left(f^{p}\right)\right\}
\end{aligned}
$$

so that

$$
\mu_{0} e^{-\sigma_{p} s}\left|\nabla T_{t} f\right|^{p} \leq \frac{p}{2} \eta^{2 / p} T_{s}\left(\mathcal{Q}\left[\nabla T_{t-s} f\right]\left(T_{t-s} f\right)^{p-2}\right)+\left(1-\frac{p}{2}\right) \eta^{2 /(p-2)} T_{t}\left(f^{p}\right) .
$$

Integrating from 0 to $t-\varepsilon$ and using (4.7) we get

$$
\begin{aligned}
& \frac{\mu_{0}\left(1-e^{-\sigma_{p}(t-\varepsilon)}\right)}{\sigma_{p}}\left|\nabla T_{t} f\right|^{p} \\
& \leq \frac{p}{2} \eta^{2 / p} \int_{0}^{t-\varepsilon} T_{s}\left(\mathcal{Q}\left[\nabla T_{t-s} f\right]\left(T_{t-s} f\right)^{p-2}\right) d s+\left(1-\frac{p}{2}\right) \eta^{2 /(p-2)}(t-\varepsilon) T_{t}\left(f^{p}\right) \\
& \quad=\frac{p}{2} \eta^{2 / p} \frac{T_{t-\varepsilon}\left(\left(T_{\varepsilon} f\right)^{p}\right)-\left(T_{t} f\right)^{p}}{p(p-1)}+\left(1-\frac{p}{2}\right) \eta^{2 /(p-2)}(t-\varepsilon) T_{t}\left(f^{p}\right)
\end{aligned}
$$

and then, letting $\varepsilon \rightarrow 0$,

$$
\left|\nabla T_{t} f\right|^{p} \leq \frac{\mu_{0}^{-1} \sigma_{p}}{1-e^{-\sigma_{p} t}} T_{t}\left(f^{p}\right)\left(\frac{p}{2} \eta^{2 / p} \frac{1}{p(p-1)}+\left(1-\frac{p}{2}\right) \eta^{2 /(p-2)} t\right) .
$$


Taking the optimal choice $\eta=\{p(p-1) t\}^{p(2-p) / 4}$ we finally obtain

$$
\left|\nabla T_{t} f\right|^{p} \leq \frac{\mu_{0}^{-1} \sigma_{p}}{[p(p-1)]^{p / 2} t^{p / 2-1}\left(1-e^{-\sigma_{p} t}\right)} T_{t}\left(f^{p}\right) .
$$

If $f \in C_{\mathrm{b}}(\bar{\Omega})$ and $f \geq 0$ then (4.8) follows by approximating $f$ with $f+1 / n$ and using Proposition 3.2. If $f \in C_{\mathrm{b}}(\bar{\Omega})$ then

$$
\begin{aligned}
\left|\nabla T_{t} f\right|^{p} & =\left|\nabla T_{t}\left(f^{+}-f^{-}\right)\right|^{p} \leq 2^{p-1}\left(\left|\nabla T_{t}\left(f^{+}\right)\right|^{p}+\left|\nabla T_{t}\left(f^{-}\right)\right|^{p}\right) \\
& \leq \frac{2^{p-1} \mu_{0}^{-1} \sigma_{p}}{[p(p-1)]^{p / 2} t^{p / 2-1}\left(1-e^{-\sigma_{p} t}\right)}\left(T_{t}\left(\left(f^{+}\right)^{p}\right)+T_{t}\left(\left(f^{-}\right)^{p}\right)\right) \\
& \leq \frac{2^{p} \mu_{0}^{-1} \sigma_{p}}{[p(p-1)]^{p / 2} t^{p / 2-1}\left(1-e^{-\sigma_{p} t}\right)} T_{t}\left(|f|^{p}\right)
\end{aligned}
$$

which concludes the proof.

REMARK 4.4. If $\Omega=\mathbb{R}^{N}$, we can consider the case of operators with locally Hölder continuous but nondifferentiable coefficients. In the case of differentiable coefficients, (2.2) and (2.3) are consequences of

$$
\begin{aligned}
\left|q_{i j}(x)-q_{i j}(y)\right| & \leq M \mu(x)|x-y|, & & x, y \in \Omega, \\
(F(x)-F(y)) \cdot(x-y) & \leq\left(\beta V(x)+k_{0}\right)|x-y|^{2}, & & x, y \in \Omega .
\end{aligned}
$$

Assume that the coefficients $q_{i j}$ and $F_{i}$ belong to $C_{\text {loc }}^{\alpha}\left(\mathbb{R}^{N}\right)$ and satisfy (4.9) and (4.10), and assume that $V \in C_{\text {loc }}^{1+\alpha}\left(\mathbb{R}^{N}\right)$ and it satisfies (2.4). If one considers a standard family of mollifiers $\left(\zeta_{\varepsilon}\right)_{\varepsilon>0}$ and defines $q_{i j}^{\varepsilon}=q_{i j} * \zeta_{\varepsilon}$ and $F_{i}^{\varepsilon}=F_{i} * \zeta_{\varepsilon}$, then the functions $q_{i j}^{\varepsilon}$ and $F_{i}^{\varepsilon}$ are regular and satisfy (4.9) and (4.10) with the same constants $q_{0}, \beta, k_{0}$ for all $\varepsilon>0$. Therefore $q_{i j}^{\varepsilon}$ and $F_{i}^{\varepsilon}$ satisfy (2.2) and (2.3); if $\mathcal{A}^{\varepsilon}$ denotes the operator with coefficients $q_{i j}^{\varepsilon}$, $F_{i}^{\varepsilon}$ and $V$, and if $P_{t}^{\varepsilon}$ denotes the associated semigroup, then $P_{t}^{\varepsilon}$ satisfies all the gradient estimates that we have proved, with the same constants for all $\varepsilon>0$. As $\varepsilon \rightarrow 0$ we get the gradient estimates for the semigroup $P_{t}$ associated with the operator with coefficients $q_{i j}, F_{i}$ and $V$. Indeed, from the interior estimates [13, Theorem IV.10.1] it follows that $P_{t}^{\varepsilon} f \rightarrow P_{t} f$ in $C_{\text {loc }}^{1,2}\left((0, \infty) \times \mathbb{R}^{N}\right)$.

5. Consequences and counterexamples. The aim of this section is to show on the one hand some consequences of the gradient estimates proved so far, and on the other hand, two counterexamples to some of them.

We start by giving a new formulation of the uniform gradient estimate (3.3): now we specify how the constant $C_{T}$ depends on the operator $\mathcal{A}$. This allows us to deduce a Liouville type theorem.

Corollary 5.1. Suppose that $V \equiv 0$ and (4.2) holds. Then for every $f \in C_{\mathrm{b}}(\bar{\Omega})$, 
if $\sigma_{2} \neq 0$, and

$$
\left\|\nabla P_{t} f\right\|_{\infty} \leq\left(\frac{\mu_{0}^{-1} \sigma_{2}}{2\left(1-e^{-\sigma_{2} t}\right)}\right)^{1 / 2}\|f\|_{\infty}, \quad t>0,
$$

if $\sigma_{2}=0$.

$$
\left\|\nabla P_{t} f\right\|_{\infty} \leq\left(\frac{1}{2 \mu_{0} t}\right)^{1 / 2}\|f\|_{\infty}, \quad t>0
$$

The proof is an easy consequence of Proposition 4.3 with $p=2$.

Proposition 5.2. Suppose that $V \equiv 0,(4.2)$ holds and $\sigma_{2}=2 k_{0}+\frac{1}{2} q_{0}$ $\leq 0$. If $f \in D(\mathcal{A})$ is such that $\mathcal{A} f=0$ then $f$ is constant.

Proof. Let $f \in D(\mathcal{A})$ and $\mathcal{A} f=0$. Then $P_{t} f=f$ for all $t \geq 0$. Applying Corollary 5.1 and letting $t \rightarrow \infty$ shows that $\nabla f \equiv 0$ and consequently $f$ is constant.

Now we assume that $\left(P_{t}\right)_{t \geq 0}$ extends to a contractive semigroup in $L_{\mu}^{1}(\Omega)=L^{1}(\Omega, \mu)$ for some measure $\mu$. Then, by interpolation, $P_{t}$ extends to a contractive semigroup in $L_{\mu}^{p}(\Omega)$ for all $1 \leq p<\infty$. In particular, one may take as $\mu$ the invariant measure of $P_{t}$ (when it exists), which is, by definition, a Borel probability measure such that

$$
\int_{\Omega} P_{t} f d \mu=\int_{\Omega} f d \mu
$$

for all $t \geq 0$ and $f \in C_{\mathrm{b}}(\bar{\Omega})$ (for more details see $[6]$ ).

In this situation, the pointwise gradient estimates of $\S 4$ imply global gradient estimates with respect to the $L^{p}$-norm. Moreover, if $\left(A_{p}, D\left(A_{p}\right)\right)$ denotes the generator of $P_{t}$ in $L_{\mu}^{p}(\Omega)$, we deduce that $D\left(A_{p}\right)$ embeds continuously in $W_{\mu}^{1, p}(\Omega)$.

Proposition 5.3. Suppose that $V \equiv 0$ and that (4.2) holds. For all $f \in L_{\mu}^{p}(\Omega)$, we have $P_{t} f \in W_{\mu}^{1, p}(\Omega)$ and

$$
\begin{aligned}
& \left\|\nabla P_{t} f\right\|_{p} \leq\left(\frac{\mu_{0}^{-1} \sigma_{2}}{2\left(1-e^{-\sigma_{2} t}\right)}\right)^{1 / 2}\|f\|_{p}, \quad t>0, p \geq 2, \\
& \left\|\nabla P_{t} f\right\|_{p} \leq t^{1 / p-1 / 2}\left(\frac{c_{p} \mu_{0}^{-1} \sigma_{p}}{1-e^{-\sigma_{p} t}}\right)^{1 / p}\|f\|_{p}, \quad t>0,1<p<2 .
\end{aligned}
$$

In the case where $\sigma_{p}=0, \sigma_{p} /\left(1-e^{-\sigma_{p} t}\right)$ is replaced by $1 / t$.

Proof. Fix $p \geq 2$. If $f \in C_{\mathrm{b}}(\bar{\Omega}) \cap L_{\mu}^{p}(\Omega)$ then integrating (4.5) entails that $P_{t} f \in W_{\mu}^{1, p}(\Omega)$ and it satisfies (5.1). If $f \in L_{\mu}^{p}(\Omega)$, take a sequence $\left(f_{n}\right) \subset$ $C_{\mathrm{b}}(\bar{\Omega}) \cap L_{\mu}^{p}(\Omega)$ that converges to $f$ in $L_{\mu}^{p}(\Omega)$. Writing (5.1) for $f_{n}-f_{m}$ implies that $P_{t} f_{n}$ is a Cauchy sequence in $W_{\mu}^{1, p}(\Omega)$. Therefore $P_{t} f \in W_{\mu}^{1, p}(\Omega)$ and it satisfies (5.1). The case $1<p<2$ follows similarly from (4.6). 
Corollary 5.4. Suppose that $V \equiv 0$. For all $p>1$ and $\omega>0$ there exists $C=C(p, \omega)>0$ such that

$$
\left\|\nabla P_{t} f\right\|_{p} \leq C \frac{e^{\omega t}}{\sqrt{t}}\|f\|_{p}, \quad t>0
$$

for every $f \in L_{\mu}^{p}$. Consequently, $D\left(A_{p}\right) \subset W_{\mu}^{1, p}(\Omega)$ and for all $\omega>0$ there exists $M_{\omega}>0$ such that

$$
\|\nabla u\|_{p} \leq M_{\omega}\|u\|_{p}^{1 / 2}\left\|\left(A_{p}-\omega\right) u\right\|_{p}^{1 / 2}
$$

for all $u \in D\left(A_{p}\right)$.

Proof. Fix $T>0$. From Proposition 5.3 it follows that $\left\|\nabla P_{t} f\right\|_{p} \leq$ $C_{T} t^{-1 / 2}\|f\|_{p}$ for every $\left.t \in\right] 0, T\left[\right.$ and $f \in L_{\mu}^{p}(\Omega)$ for some constant $C_{T}>0$. Therefore arguing as in Corollary 3.3 we get (5.3).

For the second statement, fix $\omega, \lambda>0$. Let $f \in C_{\mathrm{b}}(\bar{\Omega}) \cap L_{\mu}^{p}(\Omega)$ and set $u=R(\lambda+\omega, \mathcal{A}) f$. Then

$$
\nabla u(x)=\int_{0}^{\infty} e^{-(\lambda+\omega) t}\left(\nabla P_{t} f\right)(x) d t, \quad x \in \bar{\Omega} .
$$

As in Proposition 3.5, with estimate (3.10) replaced by (5.3), we deduce that

$$
\|\nabla u\|_{p} \leq M_{\omega}\|u\|_{p}^{1 / 2}\left\|\left(A_{p}-\omega\right) u\right\|_{p}^{1 / 2} .
$$

Since $C_{\mathrm{b}}(\bar{\Omega}) \cap L_{\mu}^{p}(\Omega)$ is dense in $L_{\mu}^{p}(\Omega)$, we see that $R(\lambda, \mathcal{A})\left(C_{\mathrm{b}}(\bar{\Omega}) \cap L_{\mu}^{p}(\Omega)\right)$ is a core for $\left(A_{p}, D\left(A_{p}\right)\right)$. Thus, the general case $u \in D\left(A_{p}\right)$ easily follows from the previous step by approximation.

EXAMPLE 5.5. This example shows that Proposition 4.3 fails in general for $p=1$. Consider the heat semigroup in $\mathbb{R}$,

$$
P_{t} f(x)=\frac{1}{(4 \pi t)^{1 / 2}} \int_{\mathbb{R}} e^{-(x-y)^{2} /(4 t)} f(y) d y, \quad t>0, x \in \mathbb{R},
$$

generated by the operator $\mathcal{A} u(x)=u^{\prime \prime}(x)$. The derivative is given by

$$
D P_{t} f(x)=\frac{1}{2 t(4 \pi t)^{1 / 2}} \int_{\mathbb{R}}(y-x) e^{-(x-y)^{2} /(4 t)} f(y) d y, \quad t>0, x \in \mathbb{R} .
$$

Fix $R>0$. Let $f \in C_{\mathrm{b}}(\mathbb{R})$ be such that $0 \leq f \leq 1, f(x)=0$ for $x<R-R^{-1}$ and $f(x)=1$ for $x>R$. Then

$$
\begin{aligned}
P_{t} f(0) & \leq \frac{1}{(4 \pi t)^{1 / 2}} \int_{R-R^{-1}}^{\infty} e^{-|y|^{2} /(4 t)} d y, \\
D P_{t} f(0) & \geq \frac{1}{2 t(4 \pi t)^{1 / 2}} \int_{R}^{\infty} y e^{-|y|^{2} /(4 t)} d y .
\end{aligned}
$$


Therefore

$$
D P_{t} f(0) \geq c_{R} P_{t} f(0), \quad c_{R}=\frac{1}{2 t} \int_{R}^{\infty} y e^{-|y|^{2} /(4 t)} d y\left(\int_{R-R^{-1}}^{\infty} e^{-|y|^{2} /(4 t)} d y\right)^{-1} \text {. }
$$

Using the De L'Hôspital rule, it is readily seen that $c_{R} \rightarrow \infty$ as $R \rightarrow \infty$. This means that no pointwise estimate similar to (4.5) can hold for $p=1$.

With the next counterexample we show that the gradient estimate (3.3) is not true in general without assuming the dissipativity condition (2.3). In particular we show an example in which $D(\mathcal{A})$ is not contained in $C_{\nu}^{1}(\bar{\Omega})$.

ExAmple 5.6. Consider in $\Omega=\mathbb{R}$ the operator

$$
\mathcal{A} u(x)=u^{\prime \prime}(x)+B^{\prime}(x) u^{\prime}(x)=e^{-B(x)}\left(e^{B(x)} u^{\prime}(x)\right)^{\prime}, \quad x \in \mathbb{R},
$$

where $B \in C^{2}(\mathbb{R})$ is such that $Q(x)=e^{B(x)} \int_{0}^{x} e^{-B(t)} d t \in L^{1}(\mathbb{R})$. Then in particular $e^{B} \in L^{1}(\mathbb{R})$. Let $D(\mathcal{A})=\left\{u \in C^{2}(\mathbb{R}) \cap C_{\mathrm{b}}(\mathbb{R}): \mathcal{A} u \in C_{\mathrm{b}}(\mathbb{R})\right\}$. It follows from $[22$, page 242] (see also [18, Proposition 2.1]) that $(\mathcal{A}, D(\mathcal{A})$ ) is the generator of a semigroup in $C_{\mathrm{b}}(\mathbb{R})$ having $e^{B(x)} d x$ as its invariant measure.

If $f \in C_{\mathrm{b}}(\mathbb{R})$, then the function

$$
u(x)=C_{1}+\int_{0}^{x} e^{-B(t)}\left(C_{2}+\int_{0}^{t} f(s) e^{B(s)} d s\right) d t,
$$

for arbitrary $C_{1}, C_{2} \in \mathbb{R}$, is the general solution of the equation $\mathcal{A} u=f$. Assuming that

and setting

$$
\int_{-\infty}^{\infty} f(t) e^{B(t)} d t=0
$$

$$
C_{2}=-\int_{0}^{\infty} f(t) e^{B(t)} d t=\int_{-\infty}^{0} f(t) e^{B(t)} d t
$$

we see that (5.5) gives, for $x>0$,

It follows that

$$
\begin{aligned}
u(x) & =C_{1}-\int_{0}^{x} e^{-B(t)} \int_{t}^{\infty} f(s) e^{B(s)} d s d t \\
& =C_{1}-\int_{0}^{\infty} e^{B(s)} f(s) \int_{0}^{s \wedge x} e^{-B(t)} d t d s .
\end{aligned}
$$

$$
|u(x)| \leq\left|C_{1}\right|+\|f\|_{\infty} \int_{0}^{\infty} Q(s) d s, \quad x>0
$$

which implies that $u$ is bounded at $\infty$. Similarly, since $Q \in L^{1}(]-\infty, 0[), u$ is bounded at $-\infty$. Since $\mathcal{A} u=f$, we conclude that $u \in D(\mathcal{A})$. The derivative 
of $u$ is given by

$$
u^{\prime}(x)=-e^{-B(x)} \int_{x}^{\infty} f(s) e^{B(s)} d s, \quad x \in \mathbb{R} .
$$

We claim that we can choose the functions $B$ and $f$ so that $Q \in L^{1}(\mathbb{R})$, (5.6) holds but $u^{\prime}$ is not bounded. To this end, take

$$
B(x)=-x^{4}+\log h(x)
$$

where $h \in C^{2}(\mathbb{R})$ satisfies

with

$$
\begin{cases}h(x)=\varepsilon_{n} & \text { if } x=n-\delta_{n} / 2, n \in \mathbb{N} \\ \varepsilon_{n} \leq h(x) \leq 1 & \text { if } n-\delta_{n}<x<n, n \in \mathbb{N} \\ h(x)=1 & \text { otherwise, }\end{cases}
$$

$$
\varepsilon_{n}=\frac{1}{n} e^{(n-1 / 2)^{4}-(n+1 / 2)^{4}}, \quad \delta_{n}=\frac{e^{-n^{4}}}{n^{2}} \varepsilon_{n} .
$$

As a consequence of this choice

$$
Q(x)=\left\{\begin{array}{cc}
e^{-x^{4}} \int_{0}^{x} e^{t^{4}} d t, & x<0 \\
h(x) e^{-x^{4}} \int_{0}^{x} \frac{e^{t^{4}}}{h(t)} d t, & x>0 .
\end{array}\right.
$$

Using the De L'Hôspital rule one sees that $\lim _{x \rightarrow-\infty} x^{3} Q(x)=1 / 4$ and hence that $Q \in L^{1}(]-\infty, 0[)$. If $x>0$ then

$$
\begin{aligned}
Q(x) & \leq e^{-x^{4}} \int_{0}^{x} \frac{e^{t^{4}}}{h(t)} d t \leq e^{-x^{4}} \int_{0}^{x} e^{t^{4}} d t+e^{-x^{4}} \sum_{n=1}^{[x]+1} \int_{n-\delta_{n}}^{n} \frac{e^{n^{4}}}{\varepsilon_{n}} d t \\
& \leq e^{-x^{4}} \int_{0}^{x} e^{t^{4}} d t+e^{-x^{4}} \sum_{n=1}^{\infty} \frac{\delta_{n} e^{n^{4}}}{\varepsilon_{n}}=e^{-x^{4}} \int_{0}^{x} e^{t^{4}} d t+e^{-x^{4}} \sum_{n=1}^{\infty} \frac{1}{n^{2}},
\end{aligned}
$$

which shows that $\left.Q \in L^{1}(] 0, \infty\right)$. Let $f \in C_{\mathrm{b}}(\mathbb{R})$ be such that $f(x)=1$ for all $x>0$ and (5.6) holds. Then

$$
u^{\prime}(x)=-\frac{e^{x^{4}}}{h(x)} \int_{x}^{\infty} h(t) e^{-t^{4}} d t, \quad x>0,
$$

and in particular, at $x_{n}=n-\delta_{n} / 2$,

$$
\begin{aligned}
\left|u^{\prime}\left(x_{n}\right)\right| & =\frac{e^{x_{n}^{4}}}{\varepsilon_{n}} \int_{x_{n}}^{\infty} h(t) e^{-t^{4}} d t \\
& \geq \frac{e^{(n-1 / 2)^{4}}}{\varepsilon_{n}} \int_{n}^{n+1 / 2} e^{-t^{4}} d t \geq \frac{e^{(n-1 / 2)^{4}}}{2 \varepsilon_{n}} e^{-(n+1 / 2)^{4}}=\frac{n}{2},
\end{aligned}
$$

which implies that $u^{\prime}(x)$ is unbounded at $\infty$. 
Therefore we have shown that the function $u$ belongs to $D(\mathcal{A})$ but not to $C_{\mathrm{b}}^{1}(\mathbb{R})$. This means that the gradient estimate (3.3) cannot be true. We note that in this situation the dissipativity assumption (2.3) fails since $B^{\prime \prime}$ is unbounded from above.

EXAMPLE 5.7. We now exhibit an example of a Neumann problem in a domain $\Omega$ with Lipschitz continuous boundary. In spite of the lower regularity of $\partial \Omega$, the associated semigroup satisfies the gradient estimate (4.1). Consider the Ornstein-Uhlenbeck operator

$$
\mathcal{A} u(x)=\frac{1}{2} \Delta u(x)-x \cdot \nabla u(x), \quad x \in \mathbb{R}^{N} .
$$

If we set

$$
\begin{array}{rlrl}
N\left(m, \sigma^{2}\right)(y) & =\frac{1}{(\sqrt{2 \pi} \sigma)^{N}} e^{-|y-m|^{2} /\left(2 \sigma^{2}\right)}, & & \sigma>0, m, y \in \mathbb{R}^{N}, \\
\Gamma(t, x, y) & =N\left(e^{-t} x, 1-e^{-2 t}\right)(y), & t>0, x, y \in \mathbb{R}^{N}
\end{array}
$$

then the Ornstein-Uhlenbeck semigroup in $C_{\mathrm{b}}\left(\mathbb{R}^{N}\right)$ is given by the formula

$$
\left(U_{t} f\right)(x)=\int_{\mathbb{R}^{N}} f(y) \Gamma(t, x, y) d y, \quad t>0, x \in \mathbb{R}^{N} .
$$

We fix $k \in \mathbb{N}, 0 \leq k<N$, and consider the domain $\Omega=\left\{x \in \mathbb{R}^{N}\right.$ : $\left.x_{k+1}, \ldots, x_{N}>0\right\}$. We now define the Ornstein-Uhlenbeck operator in $\Omega$ with Neumann boundary conditions. For $k+1 \leq j \leq N$ consider the reflections

$$
\theta_{j}: \mathbb{R}^{N} \rightarrow \mathbb{R}^{N}, \quad \theta_{j} x=\left(x_{1}, \ldots, x_{j-1},-x_{j}, x_{j+1}, \ldots, x_{N}\right), \quad x \in \mathbb{R}^{N},
$$

and the family

$$
\Lambda=\left\{\theta=\theta_{i_{1}} \circ \cdots \circ \theta_{i_{n}}: k+1 \leq i_{j} \leq N, i_{j}<i_{h} \text { if } j<h, 1 \leq n \leq N-k\right\} .
$$

Moreover if $f \in C_{\mathrm{b}}(\bar{\Omega})$ we define the extension $E f \in C_{\mathrm{b}}\left(\mathbb{R}^{N}\right)$ by

$$
(E f)(x)=f\left(x_{1}, \ldots, x_{k},\left|x_{k+1}\right|, \ldots,\left|x_{N}\right|\right), \quad x \in \mathbb{R}^{N} .
$$

The Ornstein-Uhlenbeck semigroup in $\Omega$ is given by the formula

$$
\left(P_{t} f\right)(x)=\left(U_{t} E f\right)(x)=\int_{\mathbb{R}^{N}}(E f)(y) \Gamma(t, x, y) d y, \quad t>0, x \in \Omega .
$$

With the change of variable $y^{\prime}=\theta y$ and using the identity $\Gamma(t, x, \theta y)=$ $\Gamma(t, \theta x, y)$ for all $\theta \in \Lambda$, we get

$$
\begin{aligned}
\left(P_{t} f\right)(x) & =\int_{\Omega} f(y)\left\{\Gamma(t, x, y)+\sum_{\theta \in \Lambda} \Gamma(t, x, \theta y)\right\} d y \\
& =\int_{\Omega} f(y)\left\{\Gamma(t, x, y)+\sum_{\theta \in \Lambda} \Gamma(t, \theta x, y)\right\} d y
\end{aligned}
$$


The Neumann boundary condition can be verified in the following way. Let $x \in \partial \Omega$ be such that $x_{j}=0$ for some $j \in\{k+1, \ldots, N\}$ and $x_{i} \neq 0$ for all $i \in\{k+1, \ldots, N\}, i \neq j$. Then the outward unit normal vector is $\nu(x)=-e_{j}$. For all $\theta \in \Lambda$ the normal derivative of the function $\Gamma(t, \theta x, y)$ is

$$
\frac{\partial}{\partial x_{j}} \Gamma(t, \theta x, y)=\frac{\left( \pm y_{j}-e^{-t} x_{j}\right) e^{-t}}{1-e^{-2 t}} \Gamma(t, \theta x, y), \quad t>0, x, y \in \Omega,
$$

where on the right hand side we have the $+\operatorname{sign}$ if $\theta$ does not contain the reflection $\theta_{j}$ and the $-\operatorname{sign}$ otherwise. Let now $\theta \in \Lambda$ be such that it does not contain the reflection $\theta_{j}$ and let $\theta^{\prime}=\theta_{j} \circ \theta \in \Lambda$; then if $x_{j}=0$ we have $\theta x=\theta^{\prime} x$ and

$$
\begin{aligned}
\frac{\partial}{\partial x_{j}} \Gamma(t, \theta x, y)+\frac{\partial}{\partial x_{j}} & \Gamma\left(t, \theta^{\prime} x, y\right) \\
= & \frac{y_{j}}{1-e^{-2 t}} \Gamma(t, \theta x, y)-\frac{y_{j}}{1-e^{-2 t}} \Gamma\left(t, \theta^{\prime} x, y\right)=0
\end{aligned}
$$

for all $t>0$ and $y \in \Omega$. Thus the Neumann boundary condition for $P_{t} f$ follows by coupling in the sum in formula (5.7) all the maps $\theta \in \Lambda$ that do not contain the reflection $\theta_{j}$ with the respective maps $\theta^{\prime}=\theta_{j} \circ \theta$. In this way all the terms of the sum are considered and the normal derivative turns out to be zero.

Since $\nabla U_{t} E f(x)=e^{-t} U_{t}(\nabla E f)(x)$ for all $x \in \mathbb{R}^{N}$, we have

$$
\left|\nabla P_{t} f(x)\right| \leq e^{-t} U_{t}(|\nabla E f|)(x)=e^{-t} P_{t}(|\nabla f|)(x), \quad t \geq 0, x \in \bar{\Omega},
$$

that is, $P_{t}$ satisfies the gradient estimate (4.1) for $p=1$ and hence for all $p \geq 1$.

\section{References}

[1] H. Amann, Dual semigroups and second order linear elliptic boundary value problems, Israel J. Math. 45 (1983), 225-254.

[2] D. Bakry, Transformations de Riesz pour les semigroupes symétriques. Seconde partie: Étude sous la condition $\Gamma_{2} \geq 0$, in: Séminaire de probabilités XIX, Lecture Notes in Math. 1123, Springer, Berlin, 1985, 145-174.

[3] D. Bakry and M. Ledoux, Lévy-Gromov's isoperimetric inequality for an infinite dimensional diffusion generator, Invent. Math. 123 (1996), 259-281.

[4] J.-M. Bony, Principe du maximum dans les espaces de Sobolev, C. R. Acad. Sci. Paris Sér. A 265 (1967), 333-336.

[5] S. Cerrai, Second Order PDE's in Finite and Infinite Dimension, Lecture Notes in Math. 1762, Springer, Berlin, 2001.

[6] G. Da Prato and J. Zabczyk, Second Order Partial Differential Equations in Hilbert Spaces, Cambridge Univ. Press, Cambridge, 2002.

[7] A. Friedman, Partial Differential Equations of Parabolic Type, Prentice-Hall, Englewood Cliffs, NJ, 1964. 
[8] S. Gallot, D. Hulin and J. Lafontaine, Riemannian Geometry, Springer, Berlin, 1990.

[9] D. Gilbarg and N. S. Trudinger, Elliptic Partial Differential Equations of Second Order, 2nd ed., Springer, Berlin, 1983.

[10] S. Itô, Fundamental Solutions of Parabolic Differential Equations and Boundary Value Problems, Japan J. Math. 27 (1957), 55-102.

[11] R. Z. Has'minskiŭ, Stochastic Stability of Differential Equations, Sijthoff and Noordhoff, Alphen aan den Rijn and Germantown, MD, 1980.

[12] N. V. Krylov, Lectures on Elliptic and Parabolic Equations in Hölder Spaces, Amer. Math. Soc., Providence, RI, 1996.

[13] O. A. Ladyzhenskaya, V. A. Solonnikov and N. N. Ural'tseva, Linear and Quasilinear Equations of Parabolic Type, Nauka, Moscow, 1967 (in Russian); English transl.: Amer. Math. Soc., Providence, RI, 1968.

[14] A. Lunardi, Analytic Semigroups and Optimal Regularity in Parabolic Problems, Birkhäuser, Basel, 1995.

[15] —, Schauder theorems for linear elliptic and parabolic problems with unbounded coefficients in $\mathbb{R}^{N}$, Studia Math. 128 (1998), 171-198.

[16] G. Metafune, D. Pallara and M. Wacker, Compactness properties of Feller semigroups, ibid. 153 (2002), 179-206.

[17] - - - 一, Feller semigroups on $\mathbb{R}^{N}$, Semigroup Forum 65 (2002), 159-205.

[18] G. Metafune and E. Priola, Some classes of non-analytic Markov semigroups, preprint.

[19] E. Priola, On a class of Markov-type semigroups in spaces of uniformly continuous and bounded functions, Studia Math. 136 (1999), 271-295.

[20] Z. Qian, A gradient estimate on a manifold with convex boundary, Proc. Roy. Soc. Edinburgh Sect. A 127 (1997), 171-179.

[21] D. W. Stroock and S. R. S. Varadhan, Multidimensional Diffusion Processes, Springer, Berlin, 1979.

[22] S. R. S. Varadhan, Lectures on Diffusion Problems and Partial Differential Equations, Tata Inst. of Fundamental Research, Bombay, 1980.

[23] F. Y. Wang, On estimation of the logarithmic Sobolev constant and gradient estimates of heat semigroups, Probab. Theory Related Fields 108 (1997), 87-101.

Dipartimento di Matematica

Università di Trento

38050 Povo (Trento), Italy

E-mail: bertoldi@science.unitn.it
Dipartimento di Matematica "Ennio De Giorgi"

Università di Lecce, C.P. 193

73100 Lecce, Italy

E-mail: sfornaro@ilenic.unile.it

Received March 13, 2003

Revised version February 26, 2004 